\title{
On the concrete hardness of Learning with Errors
}

\author{
Martin R. Albrecht ${ }^{1}$, Rachel Player ${ }^{1}$, and Sam Scott ${ }^{1}$ \\ Information Security Group, Royal Holloway, University of London
}

\begin{abstract}
The Learning with Errors (LWE) problem has become a central building block of modern cryptographic constructions. This work collects and presents hardness results for concrete instances of LWE. In particular, we discuss algorithms proposed in the literature and give the expected resources required to run them. We consider both generic instances of LWE as well as small secret variants. Since for several methods of solving LWE we require a lattice reduction step, we also review lattice reduction algorithms and use a refined model for estimating their running times. We also give concrete estimates for various families of LWE instances, provide a Sage module for computing these estimates and highlight gaps in the knowledge about algorithms for solving the Learning with Errors problem.
\end{abstract}

\section{Warning (August 2019)}

While the LWE Estimator ${ }^{1}$, which accompanies this work, has been continuously updated since its publication, this work itself has not been. We therefore caution the reader against using this work as a reference for the state of the art in assessing the cost of solving LWE or making sense of the LWE estimator. For example, we note:

- The cost of lattice reduction is estimated differently in the LWE estimator than as described in Section 3. Since commit 9d37cao, the cost of BKZ in dimension $n$ with block size $k$ is estimated as $t_{B K Z}=8 \cdot n \cdot t_{k}$, where $t_{k}$ is the cost of calling an SVP oracle in dimension $k$. The cost estimates for sieving have been updated: the classical estimate follows [BDGL16] since commit 74ee857 and a new quantum sieving estimate following [LMvdP15,Laa15] has been available since commit 509a759.

- This paper assumes that the number of LWE samples $m$ is unlimited, which may not represent the situation in practice. Bindel et al. [BBGS17] implemented an extension that enables users to specify a limit on $m$. This was incorporated into the LWE estimator at commit eb45a74.

- Many new variants [GJS15,KF15,GJMS17,EHK ${ }^{+}$18] of the BKW algorithm (see Section 5.2) have appeared since the time of writing. The LWE estimator supports Coded-BKW [GJS15] since commit c662db9.

- Albrecht [Alb17] gave a new variant of the dual attack (see Section 5.3) for situations where the secret is small and/or sparse. Estimates of this attack were incorporated into the LWE estimator at commit 6295aa5.

- Cheon et al. showed [CHHS19] that the MITM algorithm given in Section 5.1 is wrong, which results in its success probability being negligibly small in practice. Cheon et al. [CHHS19] presented an alternative MITM algorithm, and show how to combine it with Albrecht's [Alb17] dual attack to give a new hybrid dual attack. Neither the new MITM algorithm or the hybrid dual attack are incorporated in the LWE estimator as of commit 3019847.

- Section 5.5, on the primal uSVP attack, follows the analysis in [GN08,AFG14] for estimating the success of lattice reduction in solving uSVP. It has been shown [AGVW17] that a more recent estimate of Alkim et al. [ADPS16] better models the behaviour of lattice reduction in this case. This estimate has been used in the LWE estimator since commit e7c9c59.

\footnotetext{
${ }^{1}$ https://bitbucket.org/malb/lwe-estimator/
} 
- Neither the LWE estimator nor this work take "dimensions for free" [Duc18, $\left.\mathrm{ADH}^{+} 19\right]$ into account.

A more recent version of the material presented here is available in Player's thesis [Pla18]. This was up-to-date as of November 2017, and fixes many typos that are still present in this paper.

\section{Introduction}

Lattice-based cryptography. Lattice-based cryptography has become popular in recent years for several reasons. One dates back to the work of Ajtai [Ajt96] who linked the average case complexity of lattice problems to the worst case, showing that a random instance of the shortest vector problem in a lattice is hard. A second reason is its potential application in a post-quantum world, since no efficient quantum algorithms are known for lattice problems. On the other hand, problems such as factoring and discrete logarithm would no longer be hard in the presence of a quantum computer [BBD09]. A third reason is the wealth of applications of lattice-based cryptography, perhaps the most notable of which is its role in the realisation of fully homomorphic encryption by Gentry [Gen09] and in follow up works (e.g. [vDGHV10,BGV12,GHS12a]). Lattice problems have also been the basis of Public Key Encryption [Reg05], including CCA secure schemes [Pei09]; Identity Based Encryption [GPV08] and the more general Hierarchical Identity Based Encryption [CHKP12]; oblivious transfer schemes [PVW08]; Circular-Secure Encryption [ACPS09]; and Leakage-Resilient Encryption [GKPV10]. Recently, candidate constructions for multi-linear maps based on presumed hard problems in lattices have been proposed [GGH13,GGH14].

Learning with Errors. One lattice problem on whose hardness several cryptographic constructions are based is the Learning with Errors (LWE) problem [Reg05,Pei09,PW11]. LWE was introduced by Regev in [Reg05] and is provably as hard as worst-case lattice problems [Reg05,BLP+13]. It is a generalisation of the Learning Parity with Noise (LPN) problem [KMR $\left.{ }^{+} 94\right]$ into large moduli $q$.

Definition 1 (LWE [Reg09]). Let $n, q$ be positive integers, $\chi$ be a probability distribution on $\mathbb{Z}$ and $\mathbf{s}$ be a secret vector in $\mathbb{Z}_{q}^{n}$. We denote by $L_{\mathbf{s}, \chi}$ the probability distribution on $\mathbb{Z}_{q}^{n} \times \mathbb{Z}_{q}$ obtained by choosing $\mathbf{a} \in \mathbb{Z}_{q}^{n}$ uniformly at random, choosing $e \in \mathbb{Z}$ according to $\chi$ and considering it in $\mathbb{Z}_{q}$, and returning $(\mathbf{a}, c)=(\mathbf{a},\langle\mathbf{a}, \mathbf{s}\rangle+e) \in \mathbb{Z}_{q}^{n} \times \mathbb{Z}_{q}$.

Decision-LWE is the problem of deciding whether pairs $(\mathbf{a}, c) \in \mathbb{Z}_{q}^{n} \times \mathbb{Z}_{q}$ are sampled according to $L_{\mathbf{s}, \chi}$ or the uniform distribution on $\mathbb{Z}_{q}^{n} \times \mathbb{Z}_{q}$.

Search-LWE is the problem of recovering $\mathbf{s}$ from $(\mathbf{a}, c)=(\mathbf{a},\langle\mathbf{a}, \mathbf{s}\rangle+e) \in \mathbb{Z}_{q}^{n} \times \mathbb{Z}_{q}$ sampled according to $L_{\mathbf{s}, \chi}$.

Contributions. The first contribution of this survey is to gather and present algorithms available in the literature used for solving LWE. In particular, we identify three strategies for solving LWE and give the algorithms available in the literature for solving LWE via one of these strategies. While in recent years several such algorithms were proposed and analysed, most treatments of LWE do not consider these results when discussing its hardness. By providing an accessible survey on available techniques we hope to motivate research to push the state-of-the-art in this area forward.

We note that in most previous works the hardness of LWE is treated only asymptotically. Indeed, it is not uncommon to hide logarithmic and constant factors in the exponent of complexity expressions. For example, Arora and Ge [AG11] specify the complexity of their algorithm as $2^{\tilde{\mathcal{O}}\left(n^{2 \xi}\right)}$, for some $\xi$ such that $\alpha q=n^{\xi}$. While such statements - separating essential from inessential factors - allow us to understand the behaviour of various families of algorithms and of the problem in 
general, they need to be refined in order to gain insights into the concrete hardness of LWE. The importance of this could be seen, for example, when it comes to designing actual systems based on LWE. Here we must select parameters to ensure that the problem instance generated is hard with respect to a particular security parameter $\lambda$ while still keeping parameters as small as possible for performance reasons. For this we must be able to identify the fastest known way of solving LWE with that choice of parameters and be assured that this attack takes $2^{\lambda}$ operations. The second contribution of this survey is hence that where possible we provide concrete estimates for how long it takes to solve LWE.

Since for most algorithms no tight closed formulae are known expressing their complexities, the third contribution of this survey is that we provide a module for the Sage mathematics software $\left[\mathrm{S}^{+} 14\right]$ which, given the parameters of an LWE instance, outputs estimates for the concrete running time of the algorithms discussed in this survey. We also apply this estimator to various families of LWE parameters from the literature and discuss areas where the collective knowledge is limited in order to motivate further research.

Instances. To this end we need to characterise LWE instances. In this survey we always let $\chi$ be a discrete Gaussian distribution on $\mathbb{Z}$ with centre zero and width parameter $\alpha q$, denoted by $\mathcal{D}_{\mathbb{Z}, \alpha q}$. A discrete Gaussian distribution with centre $\mu$ and width parameter $\alpha q$ samples elements with a probability proportional to $\exp \left(-\pi \frac{(x-\mu)^{2}}{(\alpha q)^{2}}\right)$. The standard deviation of a continuous Gaussian with width parameter $\alpha q$ is $\sigma=\frac{\alpha q}{\sqrt{2 \pi}}$ and we roughly have this relation when we discretise, as long as $\sigma$ is bigger than the smoothing parameter $\eta_{\epsilon}(\mathbb{Z})$ of $\mathbb{Z}$ [DB13]. For ease of analysis, some works (e.g. [LP11]) treat the error terms as not too dissimilar from samples from a continuous Gaussian, and we join them in this approach whenever this occurs.

We then characterise LWE instances in the following way.

1. Typically, we have $q \approx n^{c}$ and $\alpha q=\sqrt{n}$, i.e. $\alpha \approx n^{1 / 2-c}$, for $c$ a small constant. Having $\alpha q>\sqrt{n}$ allows the reduction of GapSVP to LWE to go through [Reg09]. In particular, Regev uses $\alpha q=2 \sqrt{n}$. Intuitively this is because a step in the reduction loses a factor of $\sqrt{n}$. Furthermore, if $\alpha q<\sqrt{n}$ then Arora and Ge's algorithm is subexponential [AG11]. In this survey, we simply pick $\alpha q=\sqrt{n}$, ignoring the constant 2 as it does not affect our estimates much. In this case we may characterise the instance by $n$ (and $c$ ).

2. The most generic characterisation is by $n, \alpha, q$.

3. In some applications, the components of the secret $\mathbf{s}$ are not chosen uniformly at random from $\mathbb{Z}_{q}$ but instead we have the guarantee that they are all "small", e.g. $\mathbf{s}_{(i)} \in\{0,1\}$. In this case we characterise the instance by $n, \alpha, q, \psi$ where $\psi$ is the distribution of the $\mathbf{s}_{(i)}$.

In many applications, we are only given access to $m=\tilde{\mathcal{O}}(n)$ samples. In this case, we would characterise the instance by $m, n, \alpha, q$. However, in this work we will assume that we have access to as many samples $m$ as we require. This is a reasonable assumption because the hardness of the LWE problem itself is essentially independent of the number of samples [Reg10]. This could be explained by the result that given a fixed (polynomial) number of samples, one can generate arbitrarily many more (independent) samples, with only a slight worsening in the error [GPV08,ACPS09].

Structure. In Section 2 we give relevant tools which we will use later. In Section 3 we review lattice reduction algorithms as these will also be used later. In Section 4 we give the three main strategies for solving LWE. In Section 5 we describe the algorithms which can be used to solve LWE via a chosen strategy. In particular we consider instances of LWE characterised both by $n, \alpha, q$ and the special case $q=n^{c}, \alpha q=\sqrt{n}$. In Section 6 we concentrate on the third characterisation of LWE: those instances with a small secret. In Section 7 we apply our estimator to parameter choices from the literature. Finally, in Section 8 we make some concluding remarks. 


\section{Notation \& Tools}

Logarithms are base 2 if not stated otherwise. We write ln for the natural logarithm. We denote vectors in bold, e.g. a, and matrices in upper-case bold, e.g. A. By $\mathbf{a}_{(i)}$ we denote the $i$-th component of $\mathbf{a}$, i.e. a scalar. In contrast, $\mathbf{a}_{i}$ is the $i$-th element of a list of vectors. The concatenation of two vectors $\mathbf{a}$ and $\mathbf{b}$ is denoted $(\mathbf{a}|| \mathbf{b})$. We denote by $\langle\cdot, \cdot\rangle$ the usual dot product of two vectors and by $\langle\cdot, \cdot\rangle_{p}$ this dot product modulo $p$. We write $2 \leq \omega<3$ for any constant such that there is an algorithm which multiplies matrices in $\mathcal{O}\left(n^{\omega}\right)$ operations for sufficiently large $n$. Hence, our $\omega$ differs slightly from the definition in [BCS97] which hides logarithmic factors (cf. [Per14]). Unless stated otherwise, we use $\approx$ to denote something sufficiently close to equal that we treat it as equal for our estimates.

Since we will use lattice reduction later on, we need some basic definitions about lattices. A lattice $L$ in $\mathbb{R}^{m}$ is a discrete additive subgroup. In this survey we restrict our attention to viewing a lattice $L(\mathbf{B})$ as being generated by a (non-unique) basis $\mathbf{B}=\left\{\mathbf{b}_{0}, \ldots, \mathbf{b}_{n-1}\right\} \subset \mathbb{Z}^{m}$ of linearlyindependent integer vectors. The rank of the lattice $L$ is defined to be the rank of the basis matrix B with rows consisting of the basis vectors. If the rank equals $m$ we say that $L$ is full-rank. We are only concerned with such lattices in this work and henceforth assume that the lattices we deal with are full-rank. In addition, we are only concerned with $q$-ary lattices which are those such that $q \mathbb{Z}^{m} \subseteq L \subseteq \mathbb{Z}^{m}$. Note that every $q$-ary lattice is full-rank. Throughout, we adopt the convention that a lattice is generated by integer combinations of row vectors, to match software conventions. The volume vol $(L)$ of a full-rank lattice $L$ is the absolute value of the determinant of any basis of the lattice. The $i^{t h}$ successive minimum of a lattice, $\lambda_{i}(L)$, is the radius of the smallest ball centred at the origin containing at least $i$ linearly independent lattice vectors. The Gaussian heuristic states that $\lambda_{1}(L) \approx \sqrt{\frac{m}{2 \pi e}} \operatorname{vol}(L)^{1 / m}$. We adopt the convention that the first non-zero vector, say $\mathbf{b}_{0}$, in a reduced lattice basis is the shortest vector in the basis.

We now give four lemmas which will be useful later. The first shows that given samples from $L_{\mathbf{s}, \chi}$ we can construct LWE instances where the secret vector follows the same distribution as the error.

Lemma 1 ([ACPS09]). Let $\mathcal{D}_{\mathbb{Z}^{n}, \alpha q}$ be an $n$-dimensional extension of $\mathcal{D}_{\mathbb{Z}, \alpha q}$ to $\mathbb{Z}^{n}$ in the obvious way, i.e. each component is sampled according to $\mathcal{D}_{\mathbb{Z}, \alpha q}$. Then, given access an oracle $L_{\mathbf{s}, \chi}$ returning samples of the form $(\mathbf{a}, c)=(\mathbf{a},\langle\mathbf{a}, \mathbf{s}\rangle+e) \in \mathbb{Z}_{q}^{n} \times \mathbb{Z}_{q}$ with $\mathbf{a} \leftarrow \leftarrow_{\$} \mathcal{U}\left(\mathbb{Z}_{q}^{n}\right)$, e $\leftarrow{ }_{\$} \mathcal{D}_{\mathbb{Z}, \alpha q}$ and $\mathbf{s} \in \mathbb{Z}_{q}^{n}$, we can construct samples of the form $(\mathbf{a}, c)=(\mathbf{a},\langle\mathbf{a}, \mathbf{e}\rangle+e) \in \mathbb{Z}_{q}^{n} \times \mathbb{Z}_{q}$ with $\mathbf{a} \leftarrow \leftarrow_{\$} \mathcal{U}\left(\mathbb{Z}_{q}^{n}\right)$, e $\leftarrow{ }_{\$} \mathcal{D}_{\mathbb{Z}, \alpha q}$ and $\mathbf{e} \leftarrow_{\$} \mathcal{D}_{\mathbb{Z}^{n}, \alpha q}$ in $2 n^{2}$ operations in $\mathbb{Z}_{q}$ per sample, at the loss of $n$ samples overall and with $\mathcal{O}\left(n^{\omega}\right)$ operations for precomputation.

Proof. Take $n$ samples from $L_{\mathbf{s}, \chi}$ and write:

$$
\left(\mathbf{A}_{0}, \mathbf{c}_{0}\right)=\left(\mathbf{A}_{0}, \mathbf{A}_{0} \cdot \mathbf{s}+\mathbf{e}_{0}\right)
$$

where $\mathbf{A}_{0} \in \mathbb{Z}_{q}^{n \times n}$. With probability $\prod_{i=1}^{n}\left(q^{n}-q^{i-1}\right) / q^{n^{2}}$ this matrix is invertible. Precompute $\mathbf{A}_{0}^{-1}$ and store it; this costs $\mathcal{O}\left(n^{\omega}\right)$ operations. Now, to produce $n$ samples of the form $(\mathbf{a}, c)=$ $(\mathbf{a},\langle\mathbf{a}, \mathbf{e}\rangle+e) \in \mathbb{Z}_{q}^{n} \times \mathbb{Z}_{q}$ with $\mathbf{a} \leftarrow_{\$} \mathcal{U}\left(\mathbb{Z}_{q}^{n}\right), e \leftarrow_{\$} \mathcal{D}_{\mathbb{Z}, \alpha q}$ and $\mathbf{e} \leftarrow_{\$} \mathcal{D}_{\mathbb{Z}^{n}, \alpha q}$ we sample:

$$
\left(\mathbf{a}_{1}, \mathbf{c}_{1}\right)=\left(\mathbf{a}_{1}, \mathbf{a}_{1} \cdot \mathbf{s}+\mathbf{e}_{1}\right)
$$

from $L_{\mathbf{s}, \chi}$ and compute:

$$
\begin{aligned}
\mathbf{a}_{1} \cdot \mathbf{A}_{0}^{-1} \cdot \mathbf{c}_{0}-\mathbf{c}_{1} & =\mathbf{a}_{1} \cdot \mathbf{A}_{0}^{-1}\left(\mathbf{A}_{0} \cdot \mathbf{s}+\mathbf{e}_{0}\right)-\mathbf{a}_{1} \cdot \mathbf{s}-\mathbf{e}_{1} \\
& =\mathbf{a}_{1} \cdot \mathbf{A}_{0}^{-1} \cdot \mathbf{A}_{0} \cdot \mathbf{s}+\mathbf{a}_{1} \cdot \mathbf{A}_{0}^{-1} \mathbf{e}_{0}-\mathbf{a}_{1} \cdot \mathbf{s}-\mathbf{e}_{1} \\
& =\mathbf{a}_{1} \cdot \mathbf{s}+\mathbf{a}_{1} \cdot \mathbf{A}_{0}^{-1} \mathbf{e}_{0}-\mathbf{a}_{1} \cdot \mathbf{s}-\mathbf{e}_{1} \\
& =\mathbf{a}_{1} \cdot \mathbf{A}_{0}^{-1} \mathbf{e}_{0}-\mathbf{e}_{1} .
\end{aligned}
$$


Now, since $\mathcal{D}_{\mathbb{Z}, \alpha q}$ is symmetric and $\mathbf{A}_{0}^{-1}$ has full rank, we get that

$$
\left(\mathbf{a}_{1} \cdot \mathbf{A}_{0}^{-1}, \mathbf{a}_{1} \cdot \mathbf{A}_{0}^{-1} \mathbf{c}_{0}+\mathbf{c}_{1}\right)=\left(\mathbf{a}_{1} \cdot \mathbf{A}_{0}^{-1}, \mathbf{a}_{1} \cdot \mathbf{A}_{0}^{-1} \mathbf{e}_{0}-\mathbf{e}_{1}\right)
$$

are $n$ valid samples of the form $(\mathbf{a}, c)=(\mathbf{a},\langle\mathbf{a}, \mathbf{e}\rangle+e) \in \mathbb{Z}_{q}^{n} \times \mathbb{Z}_{q}$ with $\mathbf{a} \leftarrow_{\$} \mathcal{U}\left(\mathbb{Z}_{q}^{n}\right), e \leftarrow_{\$} \mathcal{D}_{\mathbb{Z}, \alpha q}$ and $\mathbf{e} \leftarrow_{\$} \mathcal{D}_{\mathbb{Z}^{n}, \alpha q}$. Finally, computing $\mathbf{a}_{1} \cdot \mathbf{A}_{0}^{-1}$ takes $2 n^{2}$ operations in $\mathbb{Z}_{q}$.

Given samples from $L_{\mathbf{s}, \chi}$, we may be able to construct LWE instances where the modulus is now $p$ for some particular $p<q$ by modulus switching. This technique was initially introduced to speedup homomorphic encryption [BV11] but can also be employed to reduce the cost of solving LWE in certain cases [AFFP14]. Modulus switching can be thought of as analogous to the difference between computing with single instead of double precision floating point numbers, where switching refers to opting to compute in the lower precision of a machine float. In the LWE context, for some $p<q$, modulus switching is considering an instance of LWE $(\bmod q)$ as a scaled instance of LWE $(\bmod p)$. This incurs a noise increase which is only small if $\mathbf{s}$ is small, so the technique can only be used for small secrets. The requirements on $p$ must be balanced. On the one hand, minimising $p$ will minimise the running time of most algorithms (see Section 6). On the other hand, picking $p$ too small increases the noise level, which in general leads to a higher complexity for solving LWE. Below, we choose $p$ to ensure that $\left\|\left\langle\frac{p}{q} \cdot \mathbf{a}-\left\lfloor\frac{p}{q} \cdot \mathbf{a}\right\rceil, \mathbf{s}\right\rangle\right\| \approx \frac{p}{q} \cdot\|e\|$ if $\mathbf{s}$ is small enough. This means that the new error term after modulus switching is essentially the previous error scaled. In particular, the new distribution is LWE with errors having standard deviation $\sqrt{2} \alpha p / \sqrt{2 \pi}+O(1)$. Following $\left[\mathrm{BV} 11, \mathrm{BLP}^{+} 13\right]$ we have the following lemma.

Lemma 2. Let $(\mathbf{a}, c)=(\mathbf{a},\langle\mathbf{a}, \mathbf{s}\rangle+e) \in \mathbb{Z}_{q}^{n} \times \mathbb{Z}_{q}$ be sampled from $L_{\mathbf{s}, \chi}$. Let $p \approx \sqrt{\frac{2 \pi n}{12}} \cdot \frac{\sigma_{s}}{\alpha}$, where $\sigma_{s}$ is the standard deviation of elements in the secret $\mathbf{s}$. Assume that the distribution of the secret $\mathbf{s}$ is such that it has mean 0 . If $p<q$ then

$$
(\tilde{\mathbf{a}}, \tilde{c})=\left(\left\lfloor\frac{p}{q} \cdot \mathbf{a}\right\rceil,\left\lfloor\frac{p}{q} \cdot c\right\rceil\right) \in \mathbb{Z}_{p}^{n} \times \mathbb{Z}_{p}
$$

is sample from an LWE distribution with error of standard deviation $\sqrt{2} \alpha p / \sqrt{2 \pi}+\mathcal{O}(1)$.

Proof. Consider

$$
\begin{aligned}
\left\lfloor\frac{p}{q} \cdot c\right] & =\left\lfloor\frac{p}{q}(\langle\mathbf{a}, \mathbf{s}\rangle+e) \mid\right. \\
& =\left\lfloor\left\langle\frac{p}{q} \cdot \mathbf{a}, \mathbf{s}\right\rangle_{p}+\frac{p}{q} \cdot e\right\rceil \\
& =\left\lfloor\left\langle\left\lfloor\frac{p}{q} \cdot \mathbf{a}\right\rceil, \mathbf{s}\right\rangle_{p}+\left\langle\frac{p}{q} \cdot \mathbf{a}-\left\lfloor\frac{p}{q} \cdot \mathbf{a}\right\rceil, \mathbf{s}\right\rangle_{p}+\frac{p}{q} \cdot e\right\rceil \\
& =\left\langle\left\lfloor\frac{p}{q} \cdot \mathbf{a}\right\rceil, \mathbf{s}\right\rangle_{p}+\left\langle\frac{p}{q} \cdot \mathbf{a}-\left\lfloor\frac{p}{q} \cdot \mathbf{a}\right\rceil, \mathbf{s}\right\rangle_{p}+\frac{p}{q} \cdot e+e^{\prime} \\
& =\left\langle\left\lfloor\frac{p}{q} \cdot \mathbf{a}\right\rceil, \mathbf{s}\right\rangle_{p}+e^{\prime \prime}+\frac{p}{q} \cdot e+e^{\prime}
\end{aligned}
$$

where $e^{\prime}$ is distributed in $(-0.5,0.5]$ and $e^{\prime \prime}$ is an inner product of small $n$-dimensional vectors and thus approaches a discrete Gaussian as $n$ tends to infinity. Its standard deviation is $\sqrt{n / 12} \sigma_{s}$, since $\frac{p}{q} \cdot \mathbf{a}-\left\lfloor\frac{p}{q} \cdot \mathbf{a}\right\rceil$ takes values $\in(-0.5,0.5]$. We have that $\frac{p}{q} \cdot e$ is a scaled discrete Gaussian of 
standard deviation $\frac{p}{q} \cdot \frac{\alpha q}{\sqrt{2 \pi}}=\alpha p / \sqrt{2 \pi}$. Targeting $e^{\prime \prime} \approx \frac{p}{q} \cdot e$, i.e. that the standard deviations of both distributions are the same, we get

$$
\begin{aligned}
\alpha p / \sqrt{2 \pi} & \approx \sqrt{n / 12} \sigma_{s} \\
p & \approx \sqrt{\frac{2 \pi n}{12}} \cdot \frac{\sigma_{s}}{\alpha} .
\end{aligned}
$$

Since the standard deviations of $\frac{p}{q} \cdot e$ and $e^{\prime \prime}$ are the same for this $p$, the standard deviation of the new distribution is $\sqrt{2} \alpha p / \sqrt{2 \pi}+\mathcal{O}(1)$ as claimed.

Following the literature, we assume that the new distribution output by the process described in Lemma 2 is $L_{\mathbf{s}, \mathcal{D}_{\mathbb{Z}, \sqrt{2} \alpha p+1}}$, i.e. that the error is discrete Gaussian, even for relatively small $n$.

The following lemma shows the equivalence of Decision-LWE and Search-LWE. Search to decision is trivial: if search is solved, $\mathbf{s}$ is known, so $e=c-\langle\mathbf{a}, \mathbf{s}\rangle$ can be computed. The nontrivial direction is due to Regev [Reg09], which is reproduced with proof below. Having established equivalence, whenever a method can be shown to solve Search-LWE or Decision-LWE we can speak of it solving LWE.

Lemma 3 (Lemma 4.2 in [Reg09]). Let $n \geq 1$ be some integer, $2 \leq q \leq \operatorname{poly}(n)$ be a prime, and $\chi$ be some distribution on $\mathbb{Z}_{q}$. Assume that we have access to a procedure $W$ that, for all $\mathbf{s}$, accepts with probability exponentially close to 1 on inputs from $L_{\mathbf{s}, \chi}$ and rejects with probability exponentially close to 1 on uniformly random inputs. Then, there exists an efficient algorithm $W^{\prime}$ that, given samples from $L_{\mathbf{s}, \chi}$ for some $\mathbf{s}$, outputs $\mathbf{s}$ with probability exponentially close to 1.

Proof. We show how $W^{\prime}$ finds the first component $\mathbf{s}_{(0)}$ of $\mathbf{s}$; finding the other components is similar. For any $k \in \mathbb{Z}_{q}$ consider the following transformation. Given a pair $(\mathbf{a}, c)$ as input to $W^{\prime}$, let it output the pair $(\mathbf{a}+(l, 0, \ldots, 0), c+l k)$ where $l \in \mathbb{Z}_{q}$ is chosen uniformly at random. It is easy to see that this transformation takes the uniform distribution to itself. On the other hand suppose the input pair $(\mathbf{a}, c)$ is sampled from $L_{\mathbf{s}, \chi}$. If $k=\mathbf{s}_{(0)}$ then this transformation takes $L_{\mathbf{s}, \chi}$ into itself. If $k \neq \mathbf{s}_{(0)}$ then this transformation takes $L_{\mathbf{s}, \chi}$ to the uniform distribution. There are only polynomially many (namely $q$ ) possibilities for $\mathbf{s}_{(0)}$, so we can try all of them as possible $k$ values. For each $k$ value, let the output of $W^{\prime}$ be the input to $W$. Then as $W$ can distinguish $L_{\mathbf{s}, \chi}$ from uniform, it can tell whether $k=\mathbf{s}_{(0)}$.

In what follows we will also make use of the following standard fact about the Gaussian distribution.

Lemma 4. Let $\chi$ denote the Gaussian distribution with standard deviation $\sigma$ and mean zero. Then, for all $C>0$, it holds that:

$$
\operatorname{Pr}\left[e \leftarrow_{\$} \chi:|e|>C \cdot \sigma\right] \leq \frac{2}{C \sqrt{2 \pi}} \exp \left(-C^{2} / 2\right)
$$


Proof. For $t>C \cdot \sigma$, we have $t /(C \cdot \sigma)>1$. Hence, we have:

$$
\begin{aligned}
\operatorname{Pr}\left[e \leftarrow_{\$} \chi:|e|>C \cdot \sigma\right] & =2 \cdot \int_{C \cdot \sigma}^{\infty} \frac{1}{\sigma \sqrt{2 \pi}} \exp \left(-\frac{t^{2}}{2 \sigma^{2}}\right) d t \\
& =\frac{2}{\sqrt{2 \pi}} \int_{C \cdot \sigma}^{\infty} \frac{1}{\sigma} \exp \left(-\frac{t^{2}}{2 \sigma^{2}}\right) d t \\
& \leq \frac{2}{\sqrt{2 \pi}} \int_{C \cdot \sigma}^{\infty} \frac{t}{C \sigma^{2}} \exp \left(-\frac{t^{2}}{2 \sigma^{2}}\right) d t \\
& =\frac{2}{C \sqrt{2 \pi}} \exp \left(-C^{2} / 2\right) d t .
\end{aligned}
$$

\section{Lattice Reduction Algorithms}

Many algorithms for solving LWE rely on lattice reduction as the central step. Hence, in this section we briefly review lattice reduction algorithms and discuss the current state-of-affairs in terms of estimating their running time. Since this survey is concerned with discussing algorithms for solving LWE this section is kept rather brief and the interested reader is directed to [MR09,Ngu10,Ngu11,LP11,Che13] for further details on lattices.

Lattice reduction algorithms can be viewed as a hierarchy: cases of BKZ [SE94] based on the block size parameter $k$. For $k=2$ the algorithm runs in polynomial time but the reduced basis output will only be LLL-reduced, i.e. it will only contain a short vector to within exponential factors of a shortest vector. When $k=n$, i.e the full size of the basis, then the output basis would be HKZ (Hermite-Korkine-Zolotarev) reduced. This is in some sense optimally reduced, but requires at least exponential runtime. Hence, when performing lattice reduction, one generally uses BKZ with some intermediary block size.

The quality of a basis output by a lattice reduction algorithm is characterised by the Hermite factor $\delta_{0}^{n}$, which is defined such that the shortest non-zero vector $\mathbf{b}_{0}$ in the output basis has the following property: $\left\|\mathbf{b}_{0}\right\|=\delta_{0}^{n} \operatorname{vol}(L)^{1 / n}$. We may also refer to $\delta_{0}$ itself, and call it the root-Hermite factor. We call its logarithm to base 2 the log root-Hermite factor.

\section{$3.1 \quad$ LLL}

LLL can be considered as a generalisation of the two dimensional algorithm by Lagrange and sometimes attributed to Gauss (see for example [Jou09]). The output of the Lagrange / Gauss algorithm is a basis $\left\{\mathbf{b}_{0}, \mathbf{b}_{1}\right\}$ such that $\left\|\mathbf{b}_{0}\right\| \leq\left\|\mathbf{b}_{1}\right\|$ and the Gram-Schmidt coefficient $\mu_{1,0} \leq \frac{1}{2}$. In particular, $\left\|\mathbf{b}_{0}\right\|=\lambda_{1}$ and $\left\|\mathbf{b}_{1}\right\|=\lambda_{2}$. The algorithm works by taking in a pair of vectors $\left\{\mathbf{b}_{0}, \mathbf{b}_{1}\right\}$ arranged such that $\left\|\mathbf{b}_{0}\right\| \leq\left\|\mathbf{b}_{1}\right\|$ and then setting $\mathbf{b}_{1}=\mathbf{b}_{1}-\left\lfloor\mu_{1,0}\right\rceil \mathbf{b}_{0}$, then swapping the vectors and repeating until no more changes can be made. Thus, when this terminates, we must have $\mu_{1,0} \leq \frac{1}{2}$.

To extend into higher dimensions one would like to do something similar but the optimal way to do this is not clear because of the additional choice of directions. Notice that the Gauss algorithm ensures that $\frac{\left\|\mathbf{b}_{1}\right\|}{\left\|\mathbf{b}_{0}\right\|}$ is not too small, in particular, $\frac{\left\|\mathbf{b}_{1}\right\|}{\left\|\mathbf{b}_{0}\right\|} \geq 1-\mu_{1,0}^{2} \geq \frac{3}{4}$ (see e.g. [NV10] for more details). An LLL reduced basis satisfies a relaxed general version of this: $\frac{\left\|\mathbf{b}_{i}\right\|}{\left\|\mathbf{b}_{i-1}\right\|} \geq \delta-\mu_{i, i-1}^{2}$ for some $\delta \in\left(\frac{1}{4}, 1\right)$. This relaxation, known as the Lovász condition, is necessary for polynomial runtime. A typical choice is $\delta=\frac{3}{4}$. 
More formally, a basis $\left\{\mathbf{b}_{0}, \mathbf{b}_{1}, \ldots \mathbf{b}_{n-1}\right\}$ is LLL-reduced if it satisfies the Lovász condition (for some $\delta$ ) and it is size reduced; that is, $\mu_{i, j} \leq \frac{1}{2}$ for $0 \leq j<i \leq n-1$.

Essentially, LLL works by size reducing the basis vectors pairwise, and then checking if the Lovász condition still holds; if it does not, then it swaps the current vector with the previous vector. In more detail, let the input basis be $\left\{\mathbf{b}_{0}, \ldots \mathbf{b}_{n-1}\right\}$. Starting at $i=1$ and incrementing upwards, consider $\mathbf{b}_{i}$ and size reduce with respect to $\mathbf{b}_{j}$ for $j=i-1$ down to $j=0$. Then check if $\mathbf{b}_{i}$ and $\mathbf{b}_{i-1}$ satisfy the Lovász condition. If they do, increment $i$; if not, swap them and decrement $i$ to ensure the swap has not affected the Lovász condition holding in the previous pair.

Running Time. It is well known the runtime of LLL is polynomial and indeed this was proved as it was introduced [LLL82]. In particular for an input basis where for all $i,\left\|\mathbf{b}_{i}\right\|<B$, LLL outputs an LLL-reduced basis in time $\mathcal{O}\left(n^{5+\varepsilon} \log ^{2+\varepsilon} B\right)$ (using fast integer multiplication). In more recent variants, improvements have been made. For example, one variant introduced by Nguyen and Stehlé called L2 [NS05] provably outputs an LLL-reduced basis in time $\mathcal{O}\left(n^{5+\varepsilon} \log B+n^{4+\varepsilon} \log ^{2} B\right)$ (using fast integer multiplication). That is, one that only grows quadratically in $\log B$. Heuristically, variants of LLL achieve $\mathcal{O}\left(n^{3} \log ^{2} B\right)$ [CN11].

Quality of Output. LLL theoretically achieves a Hermite factor of $\left(\frac{4}{3}\right)^{\frac{n-1}{4}}$ [LLL82]. In practice, it behaves much better and a root-Hermite factor $\delta_{0}$ of 1.0219 is reported in [GN08].

Implementations. LLL and its variants are implemented in many software packages, notably in NTL [Sho], FLINT [HJP14] and fplll [CPS13]. The latter also implements L2.

\section{$3.2 \quad$ BKZ}

The BKZ algorithm [SE94] requires an algorithm solving exact SVP in possibly smaller dimensions as a subroutine. The typical methods of doing this are computing the Voronoi cell of the lattice, sieving or enumeration [HPS11b]. Below we refer to running any of these algorithms as calling an SVP oracle.

The BKZ algorithm runs as follows, where at every stage $\mathbf{b}_{0}, \ldots, \mathbf{b}_{n-1}$ is the updated basis. The input basis is LLL reduced, and the first block is $\mathbf{b}_{0}, \ldots, \mathbf{b}_{k-1}$. Call the SVP oracle to obtain a short vector, $\mathbf{b}_{0}^{\prime}$, in the space spanned by these vectors. We now have $k+1$ vectors spanning a $k$ dimensional space, so we call LLL to obtain a new set of $k$ linearly independent vectors. The second block is made of vectors which are the projection of $\mathbf{b}_{1}, \ldots, \mathbf{b}_{k}$ onto $\left\langle\mathbf{b}_{0}\right\rangle^{\perp}$ (the space which is the span of the orthogonal complement of $\left.\mathbf{b}_{0}\right)$. Again we call the SVP oracle to obtain a short vector in this space, $\mathbf{b}_{1}^{\prime}$, which can be viewed as the projection of some $\mathbf{b}_{1}^{\prime \prime}$ in the lattice. Now we call LLL on $\mathbf{b}_{0}, \mathbf{b}_{1}, \ldots, \mathbf{b}_{k}, \mathbf{b}_{1}^{\prime \prime}$ to update the list of basis vectors. The next block is made of vectors which are the projection of $\mathbf{b}_{2}, \ldots, \mathbf{b}_{k+1}$ onto $\left\langle\mathbf{b}_{0}, \mathbf{b}_{1}\right\rangle^{\perp}$ (the space which is the span of the orthogonal complement of $\mathbf{b}_{0}$ and $\left.\mathbf{b}_{1}\right)$, and again the SVP oracle is called to obtain a short vector in this space, which can be viewed as a projected $\mathbf{b}_{2}^{\prime \prime}$; and this procedure carries on through the basis. The first $n-k+1$ blocks are all of size $k$, and then after this point each block is one vector shorter than the previous block. The output basis of this process is another LLL reduced basis, which can be treated as a new input, and the whole process continues again, until a basis passes through unchanged, at which point the algorithm terminates.

A HKZ reduced basis $\left\{\mathbf{b}_{0}, \ldots \mathbf{b}_{n-1}\right\}$ is a basis such that its Gram-Schmidt vectors $\mathbf{b}_{i}^{*}$ satisfy $\left\|\mathbf{b}_{i}^{*}\right\|=\lambda_{1}\left(\pi_{i}(L)\right)$ for $0 \leq i \leq n-1$ where $\pi_{i}(L)=\left\langle\mathbf{b}_{0}, \ldots, \mathbf{b}_{i-2}\right\rangle^{\perp}$. We can see that BKZ 
constructively achieves a basis with the following property: each block of size $k$ (e.g. $\left.\mathbf{b}_{0}, \ldots, \mathbf{b}_{k-1}\right)$, that is all the first $n-k+1$ blocks, is a HKZ reduced basis. Therefore, if $k=n$ then the whole output basis is HKZ reduced.

BKZ 2.0. Several improvements of BKZ have been suggested and their combination is often referred to as BKZ 2.0 [CN11]. These improvements are extreme pruning [GNR10], early termination, limiting the enumeration radius to the Gaussian Heuristic and local block pre-processing. Extreme pruning takes place in the enumeration subroutine, and it works by exploring only certain branches in the search tree, with the hope that a short enough vector is still found, therefore decreasing the runtime. Early termination is based on the observation that the quality of the output basis increases more dramatically in the earlier rounds of BKZ. Therefore, continuing to reduce the lattice offers diminishing returns in the basis quality, and early termination decreases the runtime while still returning a basis close to the desired quality. Local block pre-processing takes the form of running BKZ- $k^{\prime}$ with early termination for some value $k^{\prime}$ so that the local basis is more than merely LLL reduced.

Quality of Output. Assuming that the Gaussian Heuristic (GH) and the Geometric Series Assumption (GSA) [Sch03] hold for a lattice, Chen [Che13] gives a limiting value of the rootHermite factor $\delta_{0}$ achievable by BKZ as a function of the block size $k$ :

$$
\lim _{n \rightarrow \infty} \delta_{0}=\left(v_{k}^{\frac{-1}{k}}\right)^{\frac{1}{k-1}} \approx\left(\frac{k}{2 \pi e}(\pi k)^{\frac{1}{k}}\right)^{\frac{1}{2(k-1)}}
$$

where $v_{k}$ is the volume of the unit ball in dimension $k$. Experimental evidence suggests that we may apply the right hand side of (1) as an estimate for $\delta_{0}$ also when $n$ is finite.

The 'lattice rule of thumb' is often used to given an approximation for $\delta_{0}$ for a given $k$ as $\delta_{0}=k^{\frac{1}{2 k}}$. To ease analysis, this expression, in turn, is often approximated by $\delta_{0}=2^{1 / k}$ [Ste13].

We note that depending on which estimate is used vastly different relations between $k$ and $\delta_{0}$ are assumed. To illustrate this we plot predictions for $\delta_{0}$ for block sizes $50 \leq k \leq 250$ in Figure 1 .

Assuming that (1) holds, we may conclude from Figure 1 that we do not need to consider the approximation $k^{\frac{1}{2 k}}$ as it is always too pessimistic. The approximation $2^{\frac{1}{k}}$ is closer to the actually expected behaviour, but as we will show below it implies a simple sub-exponential algorithm for solving LWE via straightforward lattice reduction.

Running Time. The running time of BKZ is mainly determined by two factors: firstly, the time $t_{k}$ it takes to find shortest or short enough vectors in lattices of dimension $k$; and secondly, the number of BKZ rounds $\rho$ needed. We assume CPU clock cycles as our basic unit to abstract from CPU clock speeds. If $t_{k}$ is the number of clock cycles it takes to solve SVP in dimension $k$ we expect BKZ to take $\rho \cdot n \cdot t_{k}$ clock cycles.

SVP Oracles. As mentioned above, three main families of algorithms exist for finding shortest vectors [HPS11b]. Computing the Voronoi cell of the lattice takes about $2^{2 k+o(k)}$ operations and $2^{k+o(k)}$ memory. Sieving takes about $2^{k+o(k)}$ operations and $2^{k+o(k)}$ memory in its provable variant [ADRS14] and $2^{0.2972 k+o(k)}$ operations and memory in its heuristic variant [LdW15]. Enumeration can be implemented in a fashion requiring $2^{\mathcal{O}\left(k^{2}\right)}$ operations and poly $(k)$ memory by running enumeration on an LLL-reduced lattice (Fincke-Pohst), but can be also be done in $k^{\mathcal{O}(k)}$ operations 


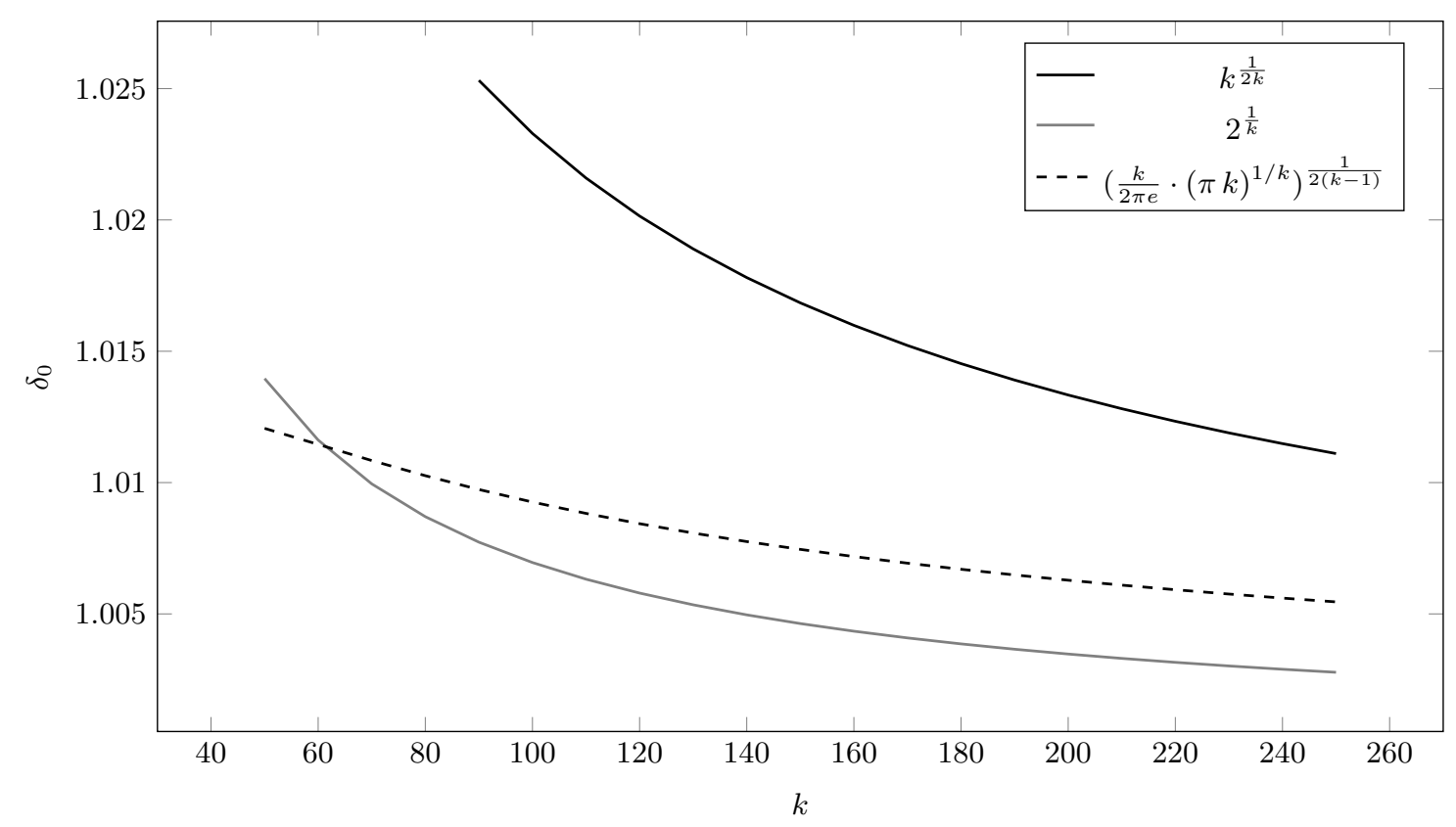

Fig. 1. Estimates for $\delta_{0}$ for BKZ- $k$.

and poly $(k)$ memory by performing heavier preprocessing on the input lattice (Kannan). Achieving $k^{\mathcal{O}(k)}$ was considered prohibitively expensive in practice until recently, but [MW15] proposed a variant which achieves $k^{\mathcal{O}(k)}$ with smaller overhead. Moreover, [Wal14] showed that preprocessing local blocks with BKZ-O $(k)$ before enumeration also reduces the complexity of BKZ- $k$ to $k^{\mathcal{O}(k)}$.

Estimating $\rho$. No closed formula for the expected number of BKZ rounds is known. The best upper bound is exponential, but after $\rho=\frac{n^{2}}{k^{2}} \log n$ many rounds, the quality of the basis is already very close to the final output [HPS11a].

Asymptotic Behaviour. Before we discuss existing estimates in the literature for the running time of BKZ, we briefly discuss the expected asymptotic behaviour of the algorithm. The 'lattice rule of thumb' puts the relation between the block size $k$ and $\delta_{0}$ as $\delta_{0}=k^{1 / 2 k}$, which implies $k / \log (k)=1 /\left(2 \log \delta_{0}\right)$. To solve this for $k$ we need the following technical lemma:

Lemma 5. For $i \geq 1$, let $g_{i}(x)=x \log \left(g_{i-1}(x)\right)$ with $g_{0}(x)=2$. Define $g_{\infty}(x)=\lim _{n \rightarrow \infty} g_{n}(x)$. If $a / \log (a)=b$ and $\log (a) \geq 1$ then

$$
a \geq g_{n}(b)
$$

for any $n \geq 0$. In particular, for $\log (a)>2, a=g_{\infty}(b)$.

Proof. For the first claim, notice that $a \geq g_{0}(b)=2$ as $\log (a) \geq 1$. Furthermore, $a \geq g_{1}(b)=b$ as $a / \log (a)=b$ so $a \geq b$. We also have $a \geq g_{2}(b)=b \log (b)$ :

$$
a=b \log a \Rightarrow a \geq b \Rightarrow \log a \geq \log b \Rightarrow a \geq b \log b
$$


For the inductive step,

$$
\begin{aligned}
\text { suppose } a & \geq g_{i}(b) \\
\Rightarrow \log a & \geq \log \left(g_{i}(b)\right) \\
a & =b \log a \Rightarrow a \geq b \log \left(g_{i}(b)\right)=g_{i+1}(b) .
\end{aligned}
$$

So by induction, we have $a \geq g_{n}(b)$. For the second claim, when $\log (a)>2, b=a / \log (a)>2$. We now prove by induction that $g_{n}(b) \geq g_{n-1}(b)$ for all $n \geq 1$. For the base case, we have $g_{1}(b)=b>2=g_{0}(b)$. For the inductive step,

$$
\begin{array}{rlrl}
\text { suppose } & g_{i}(b) & \geq g_{i-1}(b) \\
\Rightarrow \quad & \frac{g_{i}(b)}{g_{i-1}(b)} & \geq 1 \\
\Rightarrow \quad \log \left(\frac{g_{i}(b)}{g_{i-1}(b)}\right) & \geq 0 \\
\text { Now } g_{i+1}(b)-g_{i}(b)=b \log \left(\frac{g_{i}(b)}{g_{i-1}(b)}\right) & \geq 0 \\
\Rightarrow & g_{i+1}(b) & \geq g_{i}(b)
\end{array}
$$

Thus we have that $g_{n}(b)$ is an increasing sequence, and by the first claim, it is bounded above by $a$. So it is convergent and we may denote its limit by $g_{\infty}(b)$. This satisfies $g_{\infty}(b)=b \log \left(g_{\infty}(b)\right)$ and so $\frac{g_{\infty}(b)}{\log \left(g_{\infty}(b)\right)}=\frac{a}{\log (a)}$. Now, for $x \geq 4$, the function $\frac{x}{\log (x)}$ is one-to-one. Note that we have $g_{n}(b)>2$ for all $n$ so also $g_{\infty}(b)>2$.

It remains to prove that $g_{\infty}(b) \geq 4$, which implies $g_{\infty}(b)=a$. To show this, we require some further properties of the function $\frac{x}{\log (x)}$. Consider the solutions of the equation $\frac{x}{\log (x)}=2$. These are precisely $x=2$ and $x=4$. By differentiating $\frac{x}{\log (x)}$ and evaluating at these values, and with the observation that $\frac{x}{\log (x)}$ is continuous for $x>1$, we can see that $\frac{x}{\log (x)}$ takes values below 2 precisely for $2<x<4$. But, $a / \log (a)>2$. So we must be in the region $x \geq 4$. So, $\frac{x}{\log (x)}$ is injective here and we may conclude $g_{\infty}(b)=a$ as required.

By Lemma 5, we have $k \geq g_{n}\left(\frac{1}{2 \log \delta_{0}}\right)$. In particular, $k \geq g_{2}\left(\frac{1}{2 \log \delta_{0}}\right)=\frac{-\log \left(2 \log \delta_{0}\right)}{2 \log \delta_{0}}$. Ignoring constants, this expression simplifies to $\frac{-\log \left(\log \delta_{0}\right)}{\log \delta_{0}}$. Since $g_{n}\left(\frac{1}{2 \log \delta_{0}}\right)$ is an increasing sequence, we can lower bound the log of the time complexity of the BKZ algorithm as follows.

Corollary 1. The log of the time complexity for running BKZ to achieve a root-Hermite factor $\delta_{0}$ is:

$$
\begin{aligned}
& \Omega\left(\frac{\log ^{2}\left(\log \delta_{0}\right)}{\log ^{2} \delta_{0}}\right) \text { if calling the SVP oracle costs } 2^{\mathcal{O}\left(k^{2}\right)}, \\
& \Omega\left(\frac{-\log \left(\frac{-\log \log \delta_{0}}{\log \delta_{0}}\right) \log \log \delta_{0}}{\log \delta_{0}}\right) \text { if calling the SVP oracle costs } k^{\mathcal{O}(k)}, \\
& \Omega\left(\frac{-\log \log \delta_{0}}{\log \delta_{0}}\right) \text { if calling the SVP oracle costs } 2^{\mathcal{O}(k)} .
\end{aligned}
$$

Remark 1. We typically have $\log \left(\delta_{0}\right)=O(\log (n) / n)$ and so since $k / \log (k)=1 /\left(2 \log \delta_{0}\right)$ we have that $k / \log (k)=\mathcal{O}(n / \log (n))$. Since $x / \log x$ is injective for sufficiently large $x$ (e.g. $x>3$ ) we can conclude $k=O(n)$ for sufficiently large $k$ and $n$. Therefore, in most cases considered in this work, the expressions in Corollary 1 could be given as $\mathcal{O}\left(n^{2}\right), \mathcal{O}(n \log n)$ and $\mathcal{O}(n)$ because we have large $n$. 
Existing Estimates. The following estimates for the running time of BKZ exist in the literature.

- Lindner and Peikert [LP11] give an estimate for the runtime (in seconds) of BKZ as

$$
\log t_{B K Z}\left(\delta_{0}\right)=\frac{1.8}{\log \delta_{0}}-110
$$

based on experiments with the implementation of BKZ in the NTL library [Sho]. That is, improvements such as extreme pruning, early termination, and local block pre-processing were not used. To convert the estimate to a more general metric, we may notice that it was derived from experiments performed on a computer running at $2.3 \mathrm{GHz}$. We can hence convert this to clock cycles, giving a runtime of $2^{\frac{1.8}{\log \delta_{0}}-110} \cdot 2^{\log \left(2.3 \cdot 10^{9}\right)}=2^{\frac{1.8}{\log \delta_{0}}-110+\log \left(2.3 \cdot 10^{9}\right)} \approx 2^{\frac{1.8}{\log \delta_{0}}-78.9}$ clock cycles. It should be noted that this is a linear model, which does not fit the actual implementation on BKZ in the NTL library as this uses an enumeration subroutine requiring $2^{\mathcal{O}\left(k^{2}\right)}$ time. Moreover, as we will show below in Section 5.3, applying this model to predict the behaviour of BKZ leads to a subexponential algorithm for solving LWE.

- Albrecht et al. $\left[\mathrm{ACF}^{+} 15\right]$ use data points of Liu and Nguyen [LN13] to extrapolate a model similar to Lindner and Peikert's [LP11] and conclude the running time of BKZ 2.0 (in seconds) to be

$$
\log t_{B K Z}\left(\delta_{0}\right)=\frac{0.009}{\log ^{2} \delta_{0}}-27
$$

They argue that for current implementations and estimates based on them the runtime of BKZ being nonlinear in $\log \delta_{0}$ is more fitting than a linear model such as that of Lindner and Peikert. The analysis also gives a runtime in seconds on a $2.3 \mathrm{GHz}$ computer, so we can convert this into clock cycles to give a runtime of $2^{\frac{0.009}{\log ^{2} \delta_{0}}-27+\log \left(2.3 \cdot 10^{9}\right)} \approx 2^{\frac{0.009}{\log ^{2} \delta_{0}}+4.1}$. We refer to this as the delta-squared model. It should be noted, though, that the running times on which this model is based were not independently verified which limits their utility. Note that this estimate drops the $\log ^{2}\left(\log \delta_{0}\right)$ factor compared Corollary 1 and assumes that enumeration in BKZ 2.0 has a complexity of $2^{\mathcal{O}\left(k^{2}\right)}$.

- Chen and Nguyen provide a simulation algorithm for BKZ 2.0 [CN11,Che13] for arbitrarily high block size, under the assumption that each block behaves as a random basis. The authors note that this assumption may not hold for block sizes $k<50$. The algorithm takes as input the logs of the norms of the Gram-Schmidt vectors belonging to the input matrix and a block size $k$. It outputs the expected logs of the norms of the Gram-Schmidt vectors of the BKZ$k$ reduced basis as well as the number of BKZ rounds $\rho$ needed. The simulation algorithm allows one to calculate what blocksize $\mathrm{k}$ will be required to obtain the approximate $\delta_{0}$ given by BKZ 2.0 (c.f. [CN11, Table 2]). Chen and Nguyen assume the SVP is solved using a pruned enumeration and they estimate the upper bound of the cost of this, for various values of $\mathrm{k}$, in terms of number of nodes of the enumeration tree [CN11, Table 3]. The cost of BKZ is dominated by the cost of enumeration, and each round of BKZ costs "essentially $m-1$ enumeration calls" [CN11] (where $m$ is the dimension of the lattice). So the total cost of BKZ is estimated to be the number of rounds multiplied by $m-1$ multiplied by the cost of an enumeration call.

- van de Pol and Smart [vdPS13] consider the problem from the perspective of using BKZ to solve an LWE instance or some other computational problem in lattices. They assume one has a desired level of security $2^{\lambda}$ (a maximum number of operations an adversary can perform) and a given lattice dimension $m$. These are used to find the lowest $\delta_{0}$ which can be achieved in $2^{\lambda}$ operations, minimising over possible choices of the block size $k$ and the number of rounds $\rho=\rho(k, m, \lambda)$. This is in contrast to an approach where the parameters of the system correspond to a $\delta_{0}$ which then implies a certain security level. They use a table of Chen and Nguyen [CN11, Table 3] to estimate the cost of one enumeration for a given $k$ and to calculate the total number of enumerations one can perform for this $k$ (to reach the maximum of $2^{\lambda}$ operations). Note that this means they do not consider block sizes $k>250$ as 
Chen and Nguyen do not give estimates for those. Smart and van de Pol remark that $\delta_{0}$ seems to converge to a value depending only on $k$, corroborating other results in the literature. They note further that the convergence is slower in higher dimension. The approach of van de Pol and Smart was later refined in [LN14].

Estimates for $t_{k}$. In Table 1 we list estimates for solving SVP in dimension $k$ which were derived as follows.

The first row — labelled 'fplll' — was derived by calling the SVP function available in fplll 4.0.4 [CPS13] for dimensions up to 53 and by fitting $a k^{2}+b k+c$ to the logs of these averaged running times.

The second row — labelled 'enum' — was derived by fitting $a \cdot k \log (k)+b k+c$ to Table 4 in [CN12] (we note that these estimates were not independently verified) and assuming one enumeration costs 200 clock cycles as in [CN12]. We note that while BKZ 2.0 does perform local block preprocessing with BKZ- $k^{\prime}$ before calling the SVP oracle it is not clear if it as implemented in [CN12] achieves a complexity of $k^{\mathcal{O}(k)}$. This is because [CN12] does not give sufficient details to decide if preprocessing was performed with $k^{\prime}=\mathcal{O}(k)$. However, from a cryptanalytical perspective it is safer to assume it achieves this bound, which is why we chose to fit the curve as we did.

The third row is based on [Laa14] which reports a time complexity of $2^{0.3366 k+o(k)}$ asymptotically and $2^{0.45 k-19}$ seconds in practical experiments on a $2.66 \mathrm{GHz} \mathrm{CPU}$ for small $k$. Note that the leading coefficient in these experiments is bigger than 0.3366 from the asymptotic statement because of the $+o(k)$ term in the asymptotic expression. We brush over this difference and simply estimate the cost of sieving as $2^{0.3366 k+c}$ operations where we derive the additive constant $c$ from timings derived from practical experiments in [Laa14]. We note that [LdW15] does not include any experimental results. Hence, we cannot estimate when the cost hidden in the $+o(k)$ term is small enough. Indeed, estimating the cost of sieving in practice is stressed as an open problem in [LdW15].

To fit curves we used Sage's $\left[\mathrm{S}^{+} 14\right]$ find_fit function. This function calls SciPy's [JOP ${ }^{+}$] function scipy.optimize.leastsq which in turn uses MINPACK's [MGH80] Imdif.

\begin{tabular}{ll}
\hline name data source & $\log \left(t_{k}\right)$ \\
\hline fplll fplll 4.0.4 & $0.0135 k^{2}-0.2825 k+21.02$ \\
enum [CN12] & $0.270189 k \log (k)-1.0192 k+16.10$ \\
sieve [Laa14] & $0.3366 k+12.31$ \\
\hline
\end{tabular}

Table 1. Estimates for the cost in clock cycles to solve SVP in dimension $k$.

Overall. By setting $\rho=\frac{n^{2}}{k^{2}} \log (n)$, we assume that running BKZ for block size $k$ and dimension $n \operatorname{costs} \frac{n^{3}}{k^{2}} \log (n) \cdot t_{k}$ CPU cycles where $t_{k}$ is taken from Table 1 based on how the SVP oracle is instantiated.

Implementations. BKZ is implemented in NTL [Sho] and fplll [CPS13]. Neither of these implementations incorporate all techniques which are collectively known as BKZ 2.0. Hence, both implementations have a complexity of $2^{\mathcal{O}\left(k^{2}\right)}$. However, the next version of fplll implements recursive local block preprocessing with BKZ [ACPS14]. 


\subsection{Choosing the number of samples $m$}

In some of the algorithms below we will have a choice of which lattice to consider. In particular, the situation will arise where our task is to find a vector with a target norm in a lattice with a given volume vol $(L)$ but variable dimension. Given this degree of freedom, we will have to choose an optimal subdimension $m$ to perform lattice reduction on. To find this optimal subdimension we need to find $m$ such that

$$
\|\mathbf{v}\|=\delta_{0}^{m} \operatorname{vol}(L)^{1 / m}
$$

is minimised. If, as in many applications below, $\operatorname{vol}(L)=q^{n}$ this becomes $\|\mathbf{v}\|=\delta_{0}^{m} q^{n / m}$, then

$$
m=\sqrt{\frac{n \log q}{\log \delta_{0}}}
$$

is the optimal subdimension to consider [MR09]. This 'optimal subdimension' is also often heuristically chosen even where the above relation between volume and dimension does not hold. In [vdPS13] the authors choose $m$ based on the best $\delta_{0}$ which can be obtained for a given security level. In one example the dimension they choose is similar to the 'optimal subdimension'.

\section{Strategies}

In this section we discuss three strategies for solving LWE: solving Decision-LWE by finding a short vector $\mathbf{v}$ such that $\langle\mathbf{v}, \mathbf{a}\rangle=0$; solving Search-LWE by finding a short $e$ such that $\langle\mathbf{a}, \mathbf{x}\rangle=c-e$ for some unknown $\mathbf{x}$; or solving Search-LWE by finding an $\mathbf{s}^{\prime}$ such that $\left\langle\mathbf{a}, \mathbf{s}^{\prime}\right\rangle$ is close to $c$. All algorithms in Section 5 follow one of these strategies.

\subsection{Short Integer Solutions (SIS)}

To distinguish the case where $m$ samples $(\mathbf{A}, \mathbf{c})$ either: follow $L_{\mathbf{s}, \chi}$, and hence satisfy $\mathbf{c}=\mathbf{A} \mathbf{s}+\mathbf{e}$ with $\mathbf{e}_{(i)} \leftarrow_{\$} \mathcal{D}_{\mathbb{Z}, \alpha q}$; or $\mathbf{c}$ is uniformly random, we can try to find a short vector $\mathbf{v}$ such that $\mathbf{v} \cdot \mathbf{A}=0$. Expressed as a lattice problem, we aim to find a vector $\mathbf{v}$ in the scaled (by $q$ ) dual lattice of the lattice generated by $\mathbf{A}$, i.e. the lattice $L=\left\{\mathbf{w} \in \mathbb{Z}_{q}^{m} \mid \mathbf{w} \mathbf{A} \equiv 0 \bmod q\right\}$, which is exactly solving the Short Integer Solutions problem [Ajt96]. Consider $\langle\mathbf{v}, \mathbf{c}\rangle$. If $\mathbf{c}=\mathbf{A s}+\mathbf{e}$ then $\langle\mathbf{v}, \mathbf{c}\rangle=\langle\mathbf{v}, \mathbf{e}\rangle$ which follows a Gaussian distribution over $\mathbb{Z}$ considered modulo $q$. In particular, it often returns small samples as both $\mathbf{v}$ and $\mathbf{e}$ are small. On the other hand, if $\mathbf{c}$ is uniform then $\langle\mathbf{v}, \mathbf{c}\rangle$ is uniform on $\mathbb{Z}_{q}$. So we may distinguish these two cases, thus solving Decision-LWE. We must however ensure $\|\mathbf{v}\|$ is suitably short. If $\|\mathbf{v}\|$ is too large then the (Gaussian) distribution of $\langle\mathbf{v}, \mathbf{e}\rangle$ will be too flat to distinguish from random. In particular, we have the following lemma:

Lemma 6 ([LP11]). Given an LWE instance characterised by $n, \alpha, q$ and a vector $\mathbf{v}$ of length $\|\mathbf{v}\|$ in the scaled dual lattice $L=\left\{\mathbf{w} \in \mathbb{Z}_{q}^{m} \mid \mathbf{w} \mathbf{A} \equiv 0 \bmod q\right\}$, the advantage of distinguishing $\langle\mathbf{v}, \mathbf{e}\rangle$ from random is close to $\exp \left(-\pi(\|\mathbf{v}\| \cdot \alpha)^{2}\right)$.

Remark 2. For example, Stehlé [Ste13] states that a suitably short choice to distinguish $L_{\mathbf{s}, \chi}$ from random is $\|\mathbf{v}\| \cdot \alpha q \leq q$, i.e. $\|\mathbf{v}\|=1 / \alpha$. By Lemma 6 , this results in an advantage of about $1 / 23$ to distinguish correctly. 
We note that depending on the algorithm used to obtain the short vector $\mathbf{v}$, it may be advantageous to accept a longer vector as output. This decreases the distinguishing advantage $\epsilon$, but then running the algorithm about $1 / \epsilon^{2}$ times will achieve a success probability close to 1 by the Chernoff bound [Che52]. This may be faster than the alternative, which uses fewer vectors (runs of the algorithm) at a higher success probability, but takes significantly longer to obtain these shorter vectors.

Corollary 2. To obtain a probability $\epsilon$ of success in solving an LWE instance parametrised by $n$, $q$ and $\alpha$ via the SIS strategy, we require a vector $\mathbf{v}$ of norm $\|\mathbf{v}\|=\frac{1}{\alpha} \sqrt{\ln \left(\frac{1}{\epsilon}\right) / \pi}$.

Methods of finding a short vector in the dual lattice, or in a lattice generally, will be described in the sections below. For ease of exposition we let $f(\epsilon)$ denote $\sqrt{\ln \left(\frac{1}{\epsilon}\right) / \pi}$.

\subsection{Bounded Distance Decoding (BDD)}

Given $m$ samples $(\mathbf{A}, \mathbf{c}=\mathbf{A} \mathbf{s}+\mathbf{e})$ following $L_{\mathbf{s}, \chi}$ we may observe that $\mathbf{c}$ is close to a linear combination of the columns of $\mathbf{A}$. Furthermore, since the noise is Gaussian, almost all of the noise is within, say, three times the standard deviation (that is, $\frac{3 \alpha q}{\sqrt{2 \pi}}$ ) from 0. Consider the lattice spanned by the columns of $\mathbf{A}$. We can see that $\mathbf{c}$ is a point which is bounded in distance from a lattice point $\mathbf{w}=\mathbf{A s}$. Hence, we may view the LWE instance as a Bounded Distance Decoding (BDD) problem instance in this lattice. This problem is as follows: given a basis of a lattice, a target vector, and a bound on the distance from the target to the lattice, find a lattice vector within that bound of the target vector. In this case, our solution to the BDD problem would be the lattice point $\mathbf{w}$, from which we may then use linear algebra to recover $\mathbf{s}$ and therefore solve Search-LWE. (In the event $\mathbf{A}$ is not invertible, call for more samples until it is.)

Again, depending on the algorithm, it may be advantageous to accept a lower success probability $\epsilon$. Then, approximately $\log \left(1-\epsilon^{\prime}\right) / \log (1-\epsilon)$ iterations will achieve a success probability close to the target $\epsilon^{\prime}$, since $\epsilon^{\prime}=1-(1-\epsilon)^{m}$.

\subsection{Solving for $\mathrm{s}$}

A variant of the previous strategy is to search for a suitable $\mathbf{s}$ directly such that $\|\mathbf{A s}-\mathbf{c}\|$ is small. This literally solves Search-LWE. While this and the previous technique are related by simple linear algebra, i.e. knowing e trivially allows to recover $\mathbf{s}$ and vice versa, they differ in which of $\mathbf{e}$ or $\mathbf{s}$ they target. For example, the Arora-Ge algorithm (cf. Section 5.6) directly recovers s.

\section{Algorithms}

\subsection{Exhaustive Search}

Exhaustive search directly solves for $\mathbf{s}$ as in Section 4.3 .

Theorem 1. The time complexity of solving Search-LWE with success probability $\epsilon$ with exhaustive search is $m \cdot(2 t \alpha q+1)^{n} \cdot 2 n=2^{n \log (2 t \alpha q+1)+\log n+1+\log m}$. The memory complexity is $n$, the sample complexity is $n+m$ with

$$
m=(\log (1-\epsilon)-n \log (2 t \alpha q+1)) / \log (2 t \alpha)
$$

for some small parameter $t=\omega(\sqrt{\log n})$. 
Proof. Consider $\{-t \alpha q, \ldots, t \alpha q\}$ for $t=\omega(\sqrt{\log n})$. By Lemma 4, an LWE sample has error which falls in this range with overwhelming probability. Apply Lemma 1 to obtain an LWE instance with $\mathbf{s}_{(i)} \leftarrow_{\$} \mathcal{D}_{\mathbb{Z}, \alpha q}$, i.e. the secret is distributed the same as the error. We are therefore able to estimate the size of each component of the secret as $\left|\mathbf{s}_{(i)}\right| \leq t \alpha q$. Therefore, to check all possible secrets we must enumerate approximately $(2 t \alpha q+1)^{n}$ vectors. For each vector we perform about $2 n$ operations in $\mathbb{Z}_{q}$ when computing the inner product.

We need $n$ samples to apply Lemma 1 (if these $n$ samples do not have full rank, pick $n$ samples again from the overall set of all samples). We know that the correct $\mathbf{s}$ will produce $e_{i}=\left\langle\mathbf{a}_{i}, \mathbf{s}\right\rangle-c_{i}$ with $e_{i} \in\{-t \alpha q, \ldots, t \alpha q\}$ with overwhelming probability. Wrong guesses $\mathbf{s}^{\prime}$ will produce random elements in $\mathbb{Z}_{q}$ which land within the acceptable range with probability $\leq(\lceil 2 t \alpha q\rceil+1) / q \approx 2 t \alpha$. For the wrong guess $\mathbf{s}^{\prime}$ to pass the test it must pass for all $m$ samples, which happens with probability $(2 t \alpha)^{m}$. There are $(2 t \alpha q+1)^{n}-1$ wrong choices for $\mathbf{s}$. By the union bound, we will hence accept a false positive with probability $p_{f} \leq(2 t \alpha)^{m} \cdot(2 t \alpha q+1)^{n}$. Choosing

$$
m \geq\left(\log \left(p_{f}\right)-n \log (2 t \alpha q+1)\right) / \log (2 t \alpha)
$$

this happens with a probability $\leq p_{f}$. Picking $p_{f}=1-\epsilon$ to ensure that $p_{f}$ is sufficiently small finishes the proof.

Corollary 3. Let $q=n^{c}$ and $\alpha q=\sqrt{n}$. Then the time complexity of solving Search-LWE with success probability $\epsilon$ with exhaustive search is

$$
2^{n \log (2 t \sqrt{n}+1)+\log n+1+\log m} .
$$

The memory complexity is $n$. The sample complexity is $m+n$ with

$$
m=(\log (1-\epsilon)-n \log (2 t \sqrt{n}+1)) /\left(\left(\frac{1}{2}-c\right) \log n+\log (2 t)\right) .
$$

Remark 3. The complexity is independent of $\alpha$ and $q$ but depends on their product $\alpha q$ and $n$.

Meet-in-the-Middle As mentioned in [BG14] there is also a Meet-in-the-Middle (MITM) algorithm. MITM also directly solves for $\mathbf{s}$ as in Section 4.3. This is a time-memory trade-off and hence a faster method than a naive brute force but at the cost of an increased requirement on memory.

Theorem 2. Let an LWE instance be parametrised by $n, \alpha, q$. If there are $n+m$ samples satisfying $2 t \alpha m<1 / C$ for some constant $C>1$ and

$$
(2 t \alpha)^{m} \cdot(2 t \alpha q+1)^{n / 2}=\operatorname{poly}(n)
$$

for some small parameter $t=\omega(\sqrt{\log n})$, then there is a MITM algorithm which solves SearchLWE with non-negligible probability which runs in time

$$
\mathcal{O}\left(m(2 t \alpha q+1)^{n / 2}(2 n+(n / 2+\operatorname{poly}(n)) \cdot \log (m(2 t \alpha q+1)))\right)
$$

and requires memory $m \cdot(2 t \alpha q+1)^{n / 2}$.

Proof. Consider $\{-t \alpha q, \ldots, t \alpha q\}$ for $t=\omega(\sqrt{\log n})$. By Lemma 4, an LWE sample has error which falls in this range with overwhelming probability. Apply Lemma 1 to obtain an LWE instance with $\mathbf{s}_{(j)} \leftarrow_{\$} \mathcal{D}_{\mathbb{Z}, \alpha q}$, i.e. the secret is distributed the same as the error. This costs $n$ samples. Given $m$ 
samples $\left(\mathbf{a}_{k},\left\langle\mathbf{a}_{k}, \mathbf{s}\right\rangle+e_{k}\right)$, split $\mathbf{a}_{k}=\mathbf{a}_{k}{ }^{l} \| \mathbf{a}_{k}{ }^{r}$ in half and for each possibility $\mathbf{s}_{i}{ }^{l}$ of the first half of $\mathbf{s}$ compute the inner product of the first half of $\mathbf{a}_{k}$ and $\mathbf{s}_{i}{ }^{l}$. Let the output of guess $\mathbf{s}_{i}{ }^{l} \in \mathbb{Z}_{q}^{n / 2}$ for each of the $m$ samples be $\mathbf{u}_{\mathbf{s}_{i}{ }^{l}}=\left(\left\langle\mathbf{a}_{0}{ }^{l}, \mathbf{s}_{i}{ }^{l}\right\rangle, \ldots,\left\langle\mathbf{a}_{m-1}{ }^{l}, \mathbf{s}_{i}{ }^{l}\right\rangle\right)$. Store a table $T$ whose entries map vectors $\mathbf{u}_{\mathbf{s}_{i} l}$ to $\mathbf{s}_{i}{ }^{l}$. Generating the table costs $m \cdot 2 n \cdot(2 t \alpha q+1)^{n / 2}$ operations, since we estimate the size of each component of the secret as $\left|\mathbf{s}_{(i)}\right| \leq t \alpha q$ and hence we expect $(2 t \alpha q+1)^{n / 2}$ candidates $\mathbf{s}_{i}{ }^{l}$ and for each of these we calculate $m$ inner products. Sort the table into lexicographical ordering component-wise. This costs

$$
\mathcal{O}\left(m(2 t \alpha q+1)^{n / 2} \cdot n / 2 \cdot \log (m(2 t \alpha q+1))\right)
$$

operations.

Now, for each candidate $\mathbf{s}_{j}^{r} \in \mathbb{Z}_{q}^{n / 2}$ for the second half of the secret compute the inner product of the second half of each $\mathbf{a}_{k}$ with $\mathbf{s}_{j}{ }^{r}$ and then subtract from $c_{k}$, to obtain the vector $\mathbf{v}_{\mathbf{s}_{j}}{ }^{r}=$ $\left(c_{0}-\left\langle\mathbf{a}_{0}{ }^{r}, \mathbf{s}_{j}{ }^{r}\right\rangle, \ldots, c_{m-1}-\left\langle\mathbf{a}_{m-1}{ }^{r}, \mathbf{s}_{j}{ }^{r}\right\rangle\right)$. Query $T$ with $\mathbf{v}_{\mathbf{s}_{j}{ }^{r}}$; that is, view $T$ as a list and sort $\mathbf{v}_{\mathbf{s}_{j}}$ into this list. We can do this in $\log (|T|)=n / 2 \cdot \log (m(2 t \alpha q+1))$ operations per candidate by binary search. Since there are $(2 t \alpha q+1)^{n / 2}$ possible second halves of the secret $\mathbf{s}_{j}{ }^{r}$, the overall cost of querying is $(2 t \alpha q+1)^{n / 2} \cdot n / 2 \cdot \log (m(2 t \alpha q+1))$. When we have sorted $\mathbf{v}_{\mathbf{s}_{j}{ }^{r}}$ into the list, we return which vectors $\mathbf{u}_{\mathbf{s}_{i}{ }^{l}}$ it has fallen between, and check if they are near to $\mathbf{v}_{\mathbf{s}_{j}}{ }^{r}$ in Euclidean distance (we specify near in a moment).

If the vector $\mathbf{v}_{\mathbf{s}_{j}^{r}}$ is near to the vector $\mathbf{u}_{\mathbf{s}_{i}{ }^{l}}$ return $\mathbf{s}_{i}{ }^{l}$ and treat $\mathbf{s}_{i}{ }^{l} \| \mathbf{s}_{j}{ }^{r}$ as a candidate secret, and check if it is correct. If this process returns no secret overall, get fresh samples and start over.

If $\mathbf{s}=\mathbf{s}_{i^{\prime}}{ }^{l} \| \mathbf{s}_{i^{\prime}}{ }^{r}$ is the correct secret then $\mathbf{v}_{\mathbf{s}_{i^{\prime}}{ }^{r}}-\mathbf{u}_{\mathbf{s}_{i^{\prime}}{ }^{l}}=\left(e_{0}, \ldots, e_{m-1}\right) \bmod q$. With overwhelming probability we will have $e_{k} \in\{-t \alpha q, \ldots, t \alpha q\}$ for $0 \leq k<m$. Therefore $\left\|\mathbf{v}_{\mathbf{s}_{i^{\prime}} r}-\mathbf{u}_{\mathbf{s}_{i^{\prime}} l}\right\|=$ $\left\|\left(e_{0}, \ldots, e_{m-1}\right)\right\|=\sqrt{m}(t \alpha q)$. A candidate should be rejected if the distance between $\mathbf{u}_{\mathbf{s}_{i} l}$ and $\mathbf{v}_{\mathbf{s}_{j}} r$ is more than $\sqrt{m}(t \alpha q)$ and should be accepted otherwise.

This means that with overwhelming probability the algorithm will identify the correct secret as long as the error does not cause a wrap around $\bmod q$ on any component. That is, over the integers we have $\mathbf{v}_{\mathbf{s}_{j}{ }^{r}}-\mathbf{u}_{\mathbf{s}_{i} l}=\left(e_{0}, \ldots, e_{m-1}\right)+\left(q h_{0}, \ldots, q h_{m-1}\right)$ for constants $h_{k} \in\{-1,0,1\}$. A wrap around $\bmod q$ on one component corresponds to $h_{k}= \pm 1$ on that component, but we require that $h_{k}=0$ on all components (for our lexicographical ordering to work). The above is equivalent on each component to $c_{k}=b_{k}+e_{k}+q h_{k}$ where $b_{k}=\left\langle\mathbf{a}_{k}, \mathbf{s}\right\rangle \bmod q$. We have that $h_{k}= \pm 1$ will not occur whenever $b_{k}$ is not in a band of width $t \alpha q$ either side of $q$, but may occur otherwise. So we can bound the probability that a correct secret is rejected because of a wrap around error by the probability that at least one of the $m$ components has $b_{k} \in[0, t \alpha q] \cup[q-t \alpha q, q)$. The probability that one component has $b_{k} \in[0, t \alpha q] \cup[q-t \alpha q, q)$ is $2 t \alpha q / q=2 t \alpha$ so by the union bound the probability that at least one of the $m$ components has $b_{k} \in[0, t \alpha q] \cup[q-t \alpha q, q)$ is $\leq m \cdot 2 t \alpha$. We want to bound $m$ so that this event only happens with probability at most $1 / C$ for some constant $C$, i.e. $(2 t \alpha m)<1 / C$.

Consider now the chance of a false positive, i.e. a wrong candidate secret $\mathbf{s}_{i}{ }^{l}$ being suggested for some candidate $\mathbf{s}_{j}{ }^{r}$. Since $\mathbf{a}_{k}$ is uniformly random, for any $\mathbf{s}_{i}{ }^{l}$, we have that $\mathbf{u}_{\mathbf{s}_{i}{ }^{l}}$ is essentially a random vector where each component takes one of $q$ values. The chance of a wrong candidate $\mathbf{s}_{j}{ }^{r}$ producing a $\mathbf{v}_{\mathbf{s}_{j}}$ matching to a given $\mathbf{u}_{\mathbf{s}_{i}}$ to within distance $\sqrt{m}(t \alpha q)$ is the chance of getting to within $\pm t \alpha q$ on every component. Therefore the chance of a false positive is $((\lceil 2 t \alpha q\rceil+1) / q)^{m} \approx(2 t \alpha)^{m}$ There are $(2 t \alpha q+1)^{n / 2}-1$ wrong choices for $\mathbf{s}_{i}{ }^{l}$. We hence expect to test $(2 t \alpha)^{m} \cdot(2 t \alpha q+1)^{n / 2}$ candidates per $\mathbf{s}_{j}{ }^{r}$ and thus require

$$
(2 t \alpha)^{m} \cdot(2 t \alpha q+1)^{n / 2}=\operatorname{poly}(n) .
$$


Remark 4. If $q=n^{c}$ and $\alpha q=\sqrt{n}$, then setting $m=n$ satisfies Theorem 2 for $c \geq 2$ and $t=2 \sqrt{\log n}$.

\subsection{BKW}

The BKW (Blum, Kalai, Wasserman) algorithm was introduced in [BKW03] and shows that subexponential algorithms exist for learning parity functions in the presence of noise: the BKW algorithm solves the LPN (Learning Parity with Noise) problem in time $2^{\mathcal{O}(n / \log n)}$. BKW can be adapted to solve LWE [Reg09] and the complexity of this has been studied in $\left[\mathrm{ACF}^{+} 15\right]$. In particular, BKW solves LWE via the SIS strategy (cf. Section 4.1).

To solve with this strategy, given $m$ samples $(\mathbf{A}, \mathbf{c})$ following $L_{\mathbf{s}, \chi}$, we require short vectors $\mathbf{v}_{i}$ in the scaled (by $q$ ) dual lattice of the lattice generated by the rows of $\mathbf{A}$. BKW constructs these by adding elements from $a$ tables with $q^{b}$ entries each, where each table is used to find collisions on $b$ components of $\mathbf{a}$ (a row of $\mathbf{A})$.

In more detail, BKW constructs the $\mathbf{v}_{i}$ as follows. Given a sample $\mathbf{a}$, BKW splits the $n$ components into $a$ blocks each of width $b$. There are $a$ stages of the algorithm in which the algorithm creates tables by searching for collisions in the appropriate $b$ coefficients of $\mathbf{a}$. In the first stage after an appropriate number of samples we obtain two vectors which agree on $\mathbf{a}_{(0)}, \ldots, \mathbf{a}_{(b-1)}$. The algorithm will then take these and subtract them producing a row with $\mathbf{a}_{(0)}=\cdots=\mathbf{a}_{(b-1)}=0$ which is stored for use in the next stage (considering $\left.\mathbf{a}_{(b)}, \ldots, \mathbf{a}_{(2 b-1)}\right)$.

The $\mathbf{v}_{i}$ are of length $\sqrt{2^{a}}$. In the first stage, suppose we find a collision with the first $b$ components. Adding those vectors clearing the first $b$ components in a produces a $\mathbf{v}_{i}$ candidate of length $\sqrt{2}$ as we are adding two vectors. Moving on to the next stage, two such vectors are added to clear the next $b$ columns, resulting in a $\mathbf{v}_{i}$ candidate of length $\sqrt{2^{2}}$, and so on for all $a$ stages.

The algorithm maintains $a$ tables of size $q^{b}$ where $b=n / a$ and its running time is typically dominated by this magnitude. In general, we have the following complexity for solving DecisionLWE with BKW. ${ }^{2}$

Theorem $3\left(\left[\mathrm{ACF}^{+} \mathbf{1 5}\right]\right)$. Let $\left(\mathbf{a}_{i}, c_{i}\right)$ be samples following $L_{\mathbf{s}, \chi}$ or a uniform distribution on $\mathbb{Z}_{q}^{n} \times \mathbb{Z}_{q}, 0<b \leq n$ be a parameter, $0<\epsilon<1$ the targeted success rate and $a=n / b$ the addition depth. Then, the expected cost of the BKW algorithm to distinguish $L_{\mathbf{s}, \chi}$ from random with success probability $\epsilon$ is

$$
\begin{aligned}
\left(\frac{q^{b}-1}{2}\right) & \cdot\left(\frac{a(a-1)}{2} \cdot(n+1)-\frac{b a(a-1)}{4}\right) \\
& -\frac{b}{6}\left(\frac{q^{b}-1}{2}\right)\left((a-1)^{3}+\frac{3}{2}(a-1)^{2}+\frac{1}{2}(a-1)\right)
\end{aligned}
$$

additions/subtractions in $\mathbb{Z}_{q}$ to produce elimination tables,

$$
m \cdot\left(\frac{a}{2} \cdot(n+2)\right) \text { with } m=\epsilon / \exp \left(-2 \pi \alpha^{2} 2^{a}\right)
$$

\footnotetext{
${ }^{2}\left[\mathrm{ACF}^{+} 15\right]$ optimistically gives $m=\epsilon / \exp \left(-\pi \alpha^{2} 2^{a}\right)$, but by the Chernoff bound we need about $1 / \exp \left(-\pi \alpha^{2} 2^{a}\right)^{2}$ samples to distinguish.
} 
additions/subtractions in $\mathbb{Z}_{q}$ to produce samples. Furthermore,

$$
a \cdot\left\lceil\frac{q^{b}}{2}\right\rceil+m
$$

calls to $L_{\mathbf{s}, \chi}$ and storage for

$$
\left(\frac{q^{b}}{2}\right) \cdot a \cdot\left(n+1-b \frac{a-1}{2}\right)
$$

elements in $\mathbb{Z}_{q}$ are needed.

To pick $a$ and $b$, recall from Remark 2 that in order to distinguish $L_{\mathbf{s}, \chi}$ from random using SIS an appropriately short choice for $\mathbf{v}_{i}$ is $\left\|\mathbf{v}_{i}\right\| \cdot \alpha q=\sqrt{2^{a}} \cdot \alpha q \leq q$ hence a suitable choice for $a$ is $a \leq \log \left(\alpha^{-2}\right)$.

Corollary $4\left(\left[\mathrm{ACF}^{+} 15\right]\right)$. Let $a=-2 \log \alpha$ and $b=n / a$. The expected cost of the BKW algorithm to distinguish $L_{\mathbf{s}, \chi}$ from random is

$$
\begin{aligned}
\left(\frac{q^{b}-1}{2}\right) \cdot\left(\frac{a(a-1)}{2} \cdot(n+1)\right) & +\operatorname{poly}(n) \leq q^{b} \cdot a^{2} n+\operatorname{poly}(n) \\
& =\mathcal{O}\left(2^{n \log q /(-2 \log \alpha)} \cdot(-2 \log \alpha)^{2} n\right)
\end{aligned}
$$

operations in $\mathbb{Z}_{q}$ Furthermore, $a \cdot\left\lceil\frac{q^{b}}{2}\right\rceil+\operatorname{poly}(n)$ calls to $L_{\mathbf{s}, \chi}$ and storage for $\left(\frac{q^{b}}{2}\right) \cdot a \cdot n$ elements in $\mathbb{Z}_{q}$ are needed.

Specialising Corollary 4 with $q=n^{c}$ and $\alpha q=\sqrt{n}$ we get:

Corollary 5. Let $q=n^{c}, \alpha q=\sqrt{n}$. Set $a=-2 \log \alpha$ and $b=n / a$. The expected cost of the BKW algorithm to distinguish $L_{\mathbf{s}, \chi}$ from random is

$$
\begin{aligned}
\left(\frac{q^{b}-1}{2}\right) \cdot\left(\frac{a(a-1)}{2} \cdot(n+1)\right)+\operatorname{poly}(n) & \leq q^{b} \cdot\left(a^{2} n\right)+\operatorname{poly}(n) \\
& =2^{\frac{n}{2-(1 / c)}} \cdot \operatorname{poly}(n)
\end{aligned}
$$

operations in $\mathbb{Z}_{q}$.

Remark 5. It is easy to see that the complexity of the BKW algorithm is determined by $n$ and $\alpha q$ and not $\alpha$ or $q$. However as $q$ grows the leading coefficient of the complexity approaches $1 / 2$ as $1 / c$ vanishes.

Note, however, that this strategy of picking $a$ and $b$ is not optimal. These choices, which produce an easy, closed form for the complexity, ensure that $m=\operatorname{poly}(n)$, which implies that almost all time is spent constructing 'elimination tables', whereas the second step of the algorithm - producing candidates for distinguishing - is very efficient. A better strategy is to balance both steps, i.e. to find $a$ and $b$ such that

$$
\left(\frac{q^{b}-1}{2}\right) \cdot\left(\frac{a(a-1)}{2} \cdot(n+1)\right)=\epsilon / \exp \left(-2 \pi \alpha^{2} 2^{a}\right) \cdot\left(\frac{a}{2} \cdot(n+2)\right) .
$$


By balancing both sides we may reduce the complexity of the BKW algorithm to $\mathcal{O}\left(2^{(c n \log (n) /(2 c \log (n)-d))}\right)=$ $2^{n /(2-o(1))}$ for some constant $d$. This can make a significant difference for picking concrete parameters.

Example 1. Choosing $n=256, q=65537$ and $\sigma=\alpha q / \sqrt{2 \pi} \approx 25.53$ we expect the cost of distinguishing $L_{\mathbf{s}, \chi}$ to be $2^{188.1}$ operations in $\mathbb{Z}_{q}$ by Corollary 4 . Balancing the two steps of the algorithm would reduce this to $2^{171}$ operations in $\mathbb{Z}_{q}$.

The search variant of $\mathrm{BKW}$ algorithm as given in $\left[\mathrm{ACF}^{+} 15\right]$ was later improved in [DTV15] by using a discrete Fourier transform to recover a correct subset of components of $\mathbf{s}$. In particular, the authors of [DTV15] arrive at the following complexity result.

Theorem 4 ([DTV15]). Let an LWE instance be parametrised by $n, \alpha, q$ and let $a, b \in \mathbb{N}$ be such that $a \cdot b=n$. Let $C_{F F T}$ be the small constant in the complexity of the fast Fourier transform computation. Let $0<\epsilon<1$ be a targeted success rate and define $\epsilon^{\prime}=(1-\epsilon) / a$. For $0 \leq j \leq a-1$ let

$$
m_{j, \epsilon}=8 \cdot b \cdot \log \frac{q}{\epsilon}\left(1-\frac{2 \pi^{2} \sigma^{2}}{q^{2}}\right)^{-2^{a-j}} .
$$

The time complexity to recover the secret $\mathbf{s}$ with probability at least $\epsilon$ is $c_{1}+c_{2}+c_{3}+c_{4}$ where

$$
c_{1}=\frac{q^{b}-1}{2} \cdot\left(\frac{(a-1)(a-2)}{2}(k+1)-\frac{b}{6}(a(a-1)(a-2))\right)
$$

is the number of additions in $\mathbb{Z}_{q}$ to produce tables,

$$
c_{2}=\sum_{j=0}^{a-1} m_{j, \epsilon^{\prime}} \cdot \frac{a-1-j}{2}(k+2)
$$

is the number of additions in $\mathbb{Z}_{q}$ to recover $\mathbf{s}$,

$$
c_{3}=2\left(\sum_{j=0}^{a-1} m_{j, \epsilon^{\prime}}\right)+C_{F F T} \cdot k \cdot q^{b} \cdot \log q
$$

is the number of operations in $\mathbb{C}$ to prepare and compute the discrete Fourier transforms, and

$$
c_{4}=(a-1)(a-2) \cdot b \cdot \frac{q^{b}-1}{2}
$$

is the number of operations in $\mathbb{Z}_{q}$ for back substitution. Furthermore we require $(a-1) \frac{q^{b}-1}{2}+m_{0, \epsilon}$ calls to $L_{\mathbf{s}, \chi}$ and storage for

$$
\left(\frac{q^{b}-1}{2}(a-1)\left(k+1-b \frac{a-2}{2}\right)+m_{0, \epsilon}\right)
$$

elements in $\mathbb{Z}_{q}$ and $q^{b}$ elements in $\mathbb{C}$.

A reference implementation of the BKW algorithm for $\mathrm{LWE}$ as described in $\left[\mathrm{ACF}^{+} 15\right]$ is available as [Alb13]. 


\subsection{Using Lattice Reduction To Distinguish}

Lattice reduction is another means to find short vectors in the scaled dual lattice, enabling us to solve LWE via the SIS strategy. Again, we consider the scaled dual lattice $L=\left\{\mathbf{w} \in \mathbb{Z}_{q}^{m} \mid \mathbf{w} \mathbf{A} \equiv 0\right.$ $\bmod q\}$. To construct this lattice from a given $\mathbf{A} \in \mathbb{Z}_{q}^{m \times n}$ : compute a basis $\mathbf{B}$ for the nullspace of $\mathbf{A}^{T}$ over $\mathbb{Z}_{q}$, lift to $\mathbb{Z}$ and extend by $q \mathbf{I} \in \mathbb{Z}^{m \times m}$ to make it $q$-ary and compute a basis for $L$. The lattice $L$ has dimension $m$, and with high probability rank $m$ and volume $\operatorname{vol}(L)=q^{n}$ [MR09].

By our convention lattice reduction will return the shortest non-zero vector $\mathbf{b}_{0}$ it found as the first vector of a reduced basis, which by definition is a short vector in $L$, so that $\mathbf{b}_{0} \mathbf{A}=0 \bmod q$. Heuristically, for a good enough output basis all vectors could be used, as they will all be somewhat short, i.e. not too dissimilar in length from each other.

Lemma 7. Let an LWE instance be parametrised by $n, \alpha, q$. Any lattice reduction algorithm achieving log root-Hermite factor

$$
\log \delta_{0}=\frac{\log ^{2}\left(\alpha \frac{1}{f(\epsilon)}\right)}{4 n \log q}
$$

can distinguish $L_{\mathbf{s}, \chi}$ with probability $\epsilon$.

Proof. With high probability $\operatorname{vol}(L)=q^{n}$ and by definition the Hermite factor is $\delta_{0}^{m}=\frac{\|\mathbf{v}\|}{\operatorname{vol}(L)^{\frac{1}{m}}}$ so we have $\|\mathbf{v}\|=\delta_{0}^{m} q^{\frac{n}{m}}$. On the other hand, we require $\|\mathbf{v}\|=\frac{1}{\alpha} f(\epsilon)$ by Corollary 2. By Section 3.3 the optimal subdimension $m$ which minimises the quantity $\delta_{0}^{m} q^{\frac{n}{m}}$ is $m=\sqrt{\frac{n \log q}{\log \delta_{0}}}$. Since we assume we can choose any number of samples $m$, we always choose to use this optimal subdimension. Rearranging with this value of $m$, we obtain $\log \delta_{0}=\frac{\log ^{2}\left(\frac{1}{\alpha} f(\epsilon)\right)}{4 n \log q}=\frac{\log ^{2}\left(\alpha \frac{1}{f(\epsilon)}\right)}{4 n \log q}$ as our desired log root-Hermite factor.

Corollary 6. Given an LWE instance parametrised by $n, q=n^{c}, \alpha q=\sqrt{n}$. Any lattice reduction algorithm achieving log root-Hermite factor

$$
\log \delta_{0}=\frac{\left(\left(c-\frac{1}{2}\right) \log n+\log f(\epsilon)\right)^{2}}{4 c n \log n}
$$

can distinguish $L_{\mathbf{s}, \chi}$ with advantage $\epsilon$.

Proof.

$$
\begin{gathered}
\delta_{0}^{m} q^{\frac{n}{m}}=\|\mathbf{v}\| \\
\delta_{0}^{m} n^{\frac{c n}{m}}=n^{c-\frac{1}{2}} f(\epsilon) \\
\sqrt{\frac{c n \log n}{\log \delta_{0}}} \log \delta_{0}+\frac{c n}{\sqrt{\frac{c n \log n}{\log \delta_{0}}}} \log n=\left(c-\frac{1}{2}\right) \log n+\log f(\epsilon) \\
\frac{c n \log n \log \delta}{\log \delta_{0}}+c n \log n=\sqrt{\frac{c n \log n}{\log \delta_{0}}}\left(\left(c-\frac{1}{2}\right) \log n+\log f(\epsilon)\right) \\
2 c n \log n=\sqrt{\frac{c n \log n}{\log \delta_{0}}}\left(\left(c-\frac{1}{2}\right) \log n+\log f(\epsilon)\right)
\end{gathered}
$$




$$
\begin{aligned}
& \frac{2 c n \log n}{\left(\left(c-\frac{1}{2}\right) \log n+\log f(\epsilon)\right)}=\sqrt{\frac{c n \log n}{\log \delta_{0}}} \\
& \frac{(2 c n \log n)^{2}}{\left(\left(c-\frac{1}{2}\right) \log n+\log f(\epsilon)\right)^{2}}=\frac{c n \log n}{\log \delta_{0}} \\
& \frac{4 c n \log n}{\left(\left(c-\frac{1}{2}\right) \log n+\log f(\epsilon)\right)^{2}}=\frac{1}{\log \delta_{0}} \\
& \frac{\left(\left(c-\frac{1}{2}\right) \log n+\log f(\epsilon)\right)^{2}}{4 c n \log n}=\log \delta_{0}
\end{aligned}
$$

Remark 6. Assuming $q=n^{c}$ and $\alpha q=\sqrt{n}$ we can see that for large $q$ and hence large $c$, lattice reduction becomes easier, as we get a larger $\delta_{0}$. Contrasting this with BKW, we can see while it is somewhat competitive in time complexity with lattice reduction for small $q$, it is much worse than the latter for large $q$ as they are, for example, used in homomorphic encryption schemes [GHS12b] (cf. Section 7).

Having established the target $\delta_{0}$, we can combine it with estimates about lattice reduction running times from Section 3.2. In Table 2 we list estimates for how long it would take lattice reduction algorithms to achieve our target $\delta_{0}$ for $f(\epsilon)=1$, i.e. $\epsilon \approx 1 / 23$.

\begin{tabular}{rcc}
\hline model & block size $k$ & $\log$ clock cycles \\
\hline rule of thumb & $\frac{k}{\log k}=\frac{4 n \log q}{\log ^{2}\left(\frac{1}{\alpha}\right)}$ & $\mathcal{O}(k)$ \\
simp. rule of thumb & $\frac{4 n \log q}{\log ^{2}\left(\frac{1}{\alpha}\right)}$ & $\mathcal{O}\left(\frac{4 n \log q}{\log ^{2}\left(\frac{1}{\alpha}\right)}\right)$ \\
Lindner \& Peikert & $?$ & $\frac{7.2 n \log q}{\log ^{2}\left(\frac{1}{\alpha}\right)}-78.9$ \\
delta-squared model & $?$ & $\frac{0.144 n^{2} \log ^{2} q}{\log ^{4}\left(\frac{1}{\alpha}\right)}+4.1$ \\
\hline & $q=n^{c}, \alpha=n^{1 / 2-c}$ & \\
\hline rule of thumb $\frac{k}{\log k}=\frac{4 c n \log n}{\left(\left(c-\frac{1}{2}\right) \log n\right)^{2}}$ & $\mathcal{O}(n)$ \\
simp. rule of thumb & $\frac{4 c n \log n}{\left(\left(c-\frac{1}{2}\right) \log n\right)^{2}}$ & $\mathcal{O}\left(\frac{4 c n \log n}{\left(\left(c-\frac{1}{2}\right) \log n\right)^{2}}\right)$ \\
Lindner \& Peikert & $?$ & $\frac{7.2 c n \log n}{\left(\left(c-\frac{1}{2}\right) \log n\right)^{2}}-78.9$ \\
delta-squared model & $?$ & $\frac{0.144 c^{2} n^{2} \log n}{\left(\left(c-\frac{1}{2}\right) \log n\right)^{4}}+4.1$ \\
\hline
\end{tabular}

Table 2. Time complexity for distinguishing $L_{\mathbf{s}, \chi}$ from random with advantage $\epsilon \approx 1 / 23$ based on lattice reduction estimates from the literature.

Considering the right-most column of Table 2 it is clear that both the Lindner-Peikert model as well as the simplified lattice rule of thumb would predict a subexponential running time for solving LWE with SIS. Since this is considered not to be the case, we may discount these approximations as too optimistic.

As pointed out in Section 4.1 above, the strategy as discussed so far is not optimal. Given access to sufficiently many samples $m$ it is usually beneficial to run lattice reduction for a smaller target success probability $\epsilon^{\prime}$ and to repeat this process about $1 /\left(\epsilon^{\prime}\right)^{2}$ times to boost the overall success probability to a success probability close to 1 . 
Example 2. Setting $n=256, q=65537$ and $\sigma=\alpha q / \sqrt{2 \pi} \approx 25.53$ following [Reg09] and picking $\epsilon=0.1$ we get a target $\delta_{0}=1.0040513$ by Lemma 7 and thus $m=838$. Computing the expected number of clock cycles, we end up with the following estimates.

\begin{tabular}{rrr}
\hline model & block size $k$ & log clock cycles \\
\hline enum & 391 & 270 \\
sieve & 391 & 182 \\
\hline
\end{tabular}

In contrast, picking $\epsilon^{\prime}$ such that $1 /\left(\epsilon^{\prime}\right)^{2}$ multiplied by the solving time is minimised we get:

\begin{tabular}{|c|c|c|c|}
\hline \multicolumn{2}{|c|}{ model $\log \left(\epsilon^{\prime}\right)$} & block size $k$ & log clock cycles \\
\hline enum & -211.005232 & 267 & 204 \\
\hline sieve & -111.004801 & 304 & 164 \\
\hline
\end{tabular}

\subsection{Decoding Approach}

This approach solves LWE by solving the BDD problem (cf. [LP11]). The most basic way of solving a BDD instance is using Babai's Nearest Plane algorithm [Bab85]. This approach can be summarised as follows: let there be $m$ samples of an LWE instance parametrised by $n, \alpha, q$ so we have a set of samples $(\mathbf{A}, \mathbf{c})$. Perform lattice reduction on the lattice $L\left(\mathbf{A}^{T}\right)$ to obtain a new basis $\mathbf{B}$ for this lattice, where the quality of this basis is characterised as usual by the root-Hermite factor $\delta_{0}$. Babai's Nearest Plane algorithm works by recursively computing the closest vector on the sublattice spanned by subsets of the Gram-Schmidt vectors $\mathbf{b}_{i}^{*}$.

This recovers the vector $\mathbf{s}$ with probability

$$
\prod_{i=0}^{m-1} \operatorname{erf}\left(\frac{\left\|\mathbf{b}_{i}^{*}\right\| \sqrt{\pi}}{2 \alpha q}\right)
$$

under the assumption that sampling from the discrete Gaussian is approximately the same as sampling from a continuous Gaussian [LP11].

The probability the nearest planes algorithm finds the vector $\mathbf{s}$ is given by the probability that the error vector e lies in the parallelepiped $\mathbf{s}+\mathcal{P}\left(\mathbf{B}^{*}\right)$. So, it can be seen that in this approach the success probability is determined by the quality of the lattice reduction.

Lindner and Peikert Nearest Planes. Lindner and Peikert [LP11] suggest an alteration of Babai's algorithm, designed to widen the fundamental parallelepiped in the direction of $\mathbf{b}_{i}^{*}$ by a factor of some $d_{i} \in \mathbb{Z}_{>0}$, thereby increasing the chance of $\mathbf{e}$ falling inside it. This will find multiple solutions, which can be searched through exhaustively to find the correct solution.

This modifies the success probability to

$$
\prod_{i=0}^{m-1} \operatorname{erf}\left(\frac{d_{i} \cdot\left\|\mathbf{b}_{i}^{*}\right\| \sqrt{\pi}}{2 \alpha q}\right) .
$$

There is no obvious way to analytically determine the optimal $d_{i}$ to achieve a desired success probability. However, Lindner and Peikert suggest a simple heuristic method in which $d_{i}$ are chosen 
to maximise $\min _{1 \leq i \leq m}\left(d_{i} \cdot\left\|\mathbf{b}_{i}^{*}\right\|\right)$. This can be shown to return optimal values if we restrict our $d_{i}$ to powers of 2 only. Since $\frac{\operatorname{erf}(2 x)}{\operatorname{erf}(x)}>\frac{\operatorname{erf}(2 y)}{\operatorname{erf}(y)}$ for all $0<x<y$, clearly the optimal value is obtained by doubling $d_{i}$ whenever $d_{i} \cdot\left\|\mathbf{b}_{i}\right\|$ is minimal. Therefore, maximising the minimum of the values $d_{i} \cdot\left\|\mathbf{b}_{i}\right\|$ is optimal for $d_{i}$ powers of 2 .

Given $m, n, \alpha$ and $q$ as above, let $t_{N P}\left(\delta_{0}, \epsilon\right)=t_{\text {node }} \cdot \prod_{i=0}^{m-1} d_{i}$ such that Equation (2) is at least $\epsilon$ where $t_{\text {node }}$ is the number of clock cycles it takes to visit one node. Then the time for a decoding approach to achieve a success probability $\epsilon$ could be determined as

$$
t_{d e c}(\epsilon)=\min _{\delta_{0}}\left\{t_{B K Z}\left(\delta_{0}\right)+t_{N P}\left(\delta_{0}, \epsilon\right)\right\} .
$$

Hence, on the one hand, with a more reduced a basis, the values of $d_{i}$ can be smaller, so the Nearest Planes algorithm requires less time. On the other hand, the lattice reduction takes significantly more time for smaller approximation factors.

We note that in [LP11, Figure 4] it appears as though this quantity has not been optimised. The authors find values for $\delta_{0}$ for which the time of a decoding approach is less than an equivalent distinguishing approach (cf. Section 5.3), but these values are not necessarily optimal, i.e. the lattice reduction step and the decoding step are not always balanced.

We note that we may opt to run the algorithm many times with a lower advantage. This typically reduces the overall complexity.

Solving BDD by Enumeration: an Update (Liu, Nguyen). Liu and Nguyen [LN13] note that the Lindner Peikert algorithm (as well as Babai's) can be viewed as a form of pruned enumeration, but with a different rule to Gama, Nguyen and Regev's pruned enumeration [GNR10]. Namely, let $\mathbf{v}$ be a node and $\mathbf{t}$ be a target vector. GNR pruning keeps nodes with bounded projections whereas the Lindner Peikert algorithm keeps nodes with bounded coordinates, in particular $\left|\zeta_{i}(\mathbf{v}-\mathbf{t})\right| \leq d_{i}\left\|\mathbf{b}_{i}^{*}\right\| / 2$ where $\zeta_{i}(\mathbf{x})=\frac{\left\langle\mathbf{x}, \mathbf{b}_{i}^{*}\right\rangle}{\left\|\mathbf{b}_{i}^{*}\right\|}$. Liu and Nguyen note that this can be generalised to arbitrary bounds on coordinates, $\left|\zeta_{i}(\mathbf{v}-\mathbf{t})\right| \leq R_{i}$ for some parameters $R_{i}$ not necessarily dependent on the $\left\|\mathbf{b}_{i}^{*}\right\|$ s.

Due to these similarities between the Lindner Peikert method and pruning techniques, Liu and Nguyen implement a variant of the Lindner Peikert algorithm in the context of pruning algorithms, using arbitrary $R_{i}$. They also randomise the input basis, allowing them to repeat the algorithm multiple times, which has the result of increasing both the runtime and success probability linearly. Since we assume access to as many samples as required, we do not rely on rerandomisation when estimating complexity. These two factors result in more flexibility in tuning the parameters, and improved results for solving BDD.

However, instead of using the enumeration framework as simply a method to improve the algorithm of Lindner and Peikert, Liu and Nguyen go on to directly apply pruned enumeration to solve BDD. This follows the earlier work of Gama, Nguyen and Regev [GNR10], and uses linear pruning in which the bounds $R_{k}=\sqrt{k / m} R_{m}$ are used. Over the same parameters used in [LP11], this linear pruning is shown to improve on both the original Nearest Planes algorithm and the improved variant.

Runtime Analysis. In any lattice decoding approach, the runtime is determined by balancing the lattice reduction step against the final step which enumerates possible solutions and outputs an answer with a certain probability.

For Babai's algorithm, the runtime is determined by calculating the Gram-Schmidt orthogonalisation — which can be done with floating point arithmetic in $\mathcal{O}\left(n^{3}\right)$. If using either of the extensions 
to Babai's algorithm this is still a component, but the main factor determining the runtime is the number of points which are calculated.

Similarly, if using a form of enumeration, we are mostly interested in how many points are enumerated. Therefore, to calculate the runtime of the BDD approach, we simplify the various enumeration algorithms to two expressions: the time it takes to enumerate one point; and the success probability for a certain number of enumerations.

For example, Lindner and Peikert estimate that running Babai's algorithm once takes $t_{\text {node }}=$ $2^{-16} \cdot 2.33 \cdot 10^{9} \approx 2^{15.1}$ clock cycles, whereas [GNR10] achieve $0.94 \cdot 10^{7}$ nodes per second which is approximately $2^{-23}$ seconds per enumeration. In our estimator (cf. Section 7) we assume $t_{n o d e}=$ $2^{15.1}$.

Calculating the success probability is harder. For Nearest Planes, we can use Equation 2, but we still need to determine the optimal values for $d_{i}$ for which we do not know a closed formula. In practice, though, we can follow Lindner and Peikert's strategy of increasing $d_{i}$ one by one. For enumeration and pruning, we need to use the method as used in [LN13], which experimentally calculates the success probability by sampling.

The most significant factor affecting the success probability is the quality of the reduced basis which is provided (i.e. what value $\delta_{0}$ is achieved). For example, Babai's algorithm without a preceding lattice reduction only gives solutions up to an exponential factor. In a sense, the methods proposed here for performing a decoding approach can be seen as a way to halt the lattice reduction when it is possible to obtain a solution with a reasonable success probability, and optionally repeating to increase the probability of solving the problem. The overall runtime is then calculated by estimating the optimal time to halt the reduction and attempt to solve.

Example 3. For $n=192, q=4093, \alpha q=8.87$ [LP11] report $2^{74}$ seconds when running the attack $2^{32}$ times with advantage $2^{-32}$ and $\delta_{0}=1.0083$ whereas the randomised NP used by Liu and Nguyen is able to perform the decoding approach using a lattice reduction with $\delta_{0}=1.0077$ and $\epsilon=2^{-12}$. This lattice reduction takes $2^{65.6}$ seconds in the Lindner \& Peikert model for lattice reduction. Our estimator suggests $\epsilon=2^{-15}$ and $\delta_{0}=1.0077566$ which implies a lattice reduction cost of $2^{66.48}$ seconds also in the Lindner \& Peikert model (for compatibility).

We note that these improvements depend on balancing many parameters in an optimal way. Calculating the success probability can only be done numerically, and optimising parameters requires many computations. Our estimator (cf. Section 7) does not provide a routine for estimating the cost using [LN13] but we restrict out attention to [LP11] which gives comparable results and is easier to estimate.

\subsection{Reducing BDD to uSVP}

Albrecht, Fitzpatrick and Göpfert [AFG14] consider the complexity of solving LWE by reducing BDD to uSVP (unique Shortest Vector Problem). Formally, the $\gamma$-uSVP problem is as follows: given a lattice $L$ such that $\lambda_{2}(L)>\gamma \lambda_{1}(L)$, find a shortest nonzero vector in $L$.

To reduce BDD to uSVP Kannan's embedding technique [Kan87] is used. The idea is to embed $L(\mathbf{A})=\left\{\mathbf{A u} \mid \mathbf{u} \in \mathbb{Z}_{q}^{n}\right\}$, the lattice generated by the columns of the LWE instance (and our usual lattice for consideration when are solving with the BDD strategy), into a higher-dimensional lattice $L(\mathbf{B})$ with $\gamma$-uSVP structure. That is, $\mathbf{B}$ is constructed as

$$
\mathbf{B}=\left(\begin{array}{cc}
\tilde{\mathbf{A}} & 0 \\
\mathbf{c} & t
\end{array}\right)
$$


where $\tilde{\mathbf{A}}$ is a basis for the $q$-ary lattice spanned by the columns of $\mathbf{A}$.

Let $\mathbf{y} \in L$, for some lattice $L$, be the closest lattice point to some point $\mathbf{x}$, i.e. the point minimising $\|\mathbf{x}-\mathbf{y}\|$. We can then define the distance from $\mathbf{x}$ to the lattice $L$, dist $(\mathbf{x}, L)$, as this length. If the embedding factor $t=\operatorname{dist}(\mathbf{c}, L(\mathbf{A}))<\frac{\left.\lambda_{1}(L(\mathbf{A}))\right)}{2 \gamma}$ then $\mathrm{L}(\mathbf{B})$ contains a $\gamma$-unique shortest vector, $\mathbf{c}^{\prime}=(\mathbf{e},-t)$ [LM09], from which we can take the first $m$ components to recover $\mathbf{e}$, hence solving the BDD instance.

To solve a $\gamma$-uSVP instance, we may reduce the problem to $\kappa$-HSVP (Hermite Shortest Vector Problem). Let $\gamma=\kappa^{2}$. Lovasz [Lov86] showed that any algorithm which can solve $\kappa$-HSVP, such as a lattice reduction algorithm, can be used linearly many times to solve approximate SVP with approximation factor $\kappa^{2}$. Intuitively, a lattice with uSVP structure has one direction in which its shortest vector is somewhat shorter than all other directions. A sufficiently precise lattice reduction algorithm (for example) can produce a vector so short it must be in this special direction. More precisely, a solution to $\kappa^{2}$-approximate SVP would be a vector $\mathbf{v}$ such that $\|\mathbf{v}\| \leq \kappa^{2} \lambda_{1}(L)$. On the other hand, any vector $\mathbf{w}$ which is not the shortest (and independent of the shortest vector) satisfies $\|\mathbf{w}\| \geq \lambda_{2}(L)>\kappa^{2} \lambda_{1}(L)$. So, we must have $\mathbf{v}$ is a multiple of a shortest vector, and hence we have solved $\kappa^{2}$-uSVP. Ling et al. [LLLS11] show that whenever $\kappa>\sqrt{N}$, for $N$ the dimension of the lattice, this result can be improved. They show any algorithm solving $\kappa$-HSVP can be used to solve $\gamma$-uSVP, where $\gamma \approx \sqrt{N} \kappa$.

The above are theoretical results. In practice, an algorithm solving HSVP will solve uSVP instances where the gap is $\lambda_{2}(L)>\tau \delta_{0}^{m} \lambda_{1}(L)$ with some probability depending on $\tau$ [GN08]. The value $\tau$ is taken to be a constant, which is experimentally derived in [GN08] and which depends on both the nature of the lattices considered, the lattice reduction algorithm used and the target success rate.

To estimate the time complexity of this approach we firstly must establish $m$ and $\tau$, which depend on how we choose the embedding factor $t$. We may have $t=\|\mathbf{e}\|$ or $t<\|\mathbf{e}\|$.

Suppose firstly that $t=\|\mathbf{e}\|$. We will need the following lemma from [AFG14]. ${ }^{3}$

Lemma 8 (Lemma 2 in [AFG14]). Let $\mathbf{A} \in \mathbb{Z}_{q}^{n \times m}$, let $\alpha q>0$ and let $\epsilon^{\prime}>1$. Let $\mathbf{e} \in \mathbb{Z}_{q}^{m}$ such that each component is drawn from $\chi$ and considered mod $q$. Assuming the Gaussian heuristic for $L(\mathbf{A})$, i.e. $\lambda_{1}(L(\mathbf{A})) \geq \sqrt{\frac{m}{2 \pi e}} \operatorname{vol}(L)^{1 / m}$, and that the rows of $\mathbf{A}$ are linearly independent over $\mathbb{Z}_{q}$, we can create an embedding lattice with $\lambda_{2} / \lambda_{1}$-gap greater than

$$
\frac{\min \left\{q, q^{1-\frac{n}{m}} \sqrt{\frac{m}{2 \pi e}}\right\}}{\frac{\epsilon^{\prime} s \sqrt{m}}{\sqrt{\pi}}}
$$

with probability greater than $1-\left(\epsilon^{\prime} \cdot \exp \left(\frac{1-\epsilon^{\prime 2}}{2}\right)\right)^{m}$.

Hence, setting $t=\|\mathbf{e}\|, \mathbf{B}$ is a basis of a lattice whose gap is determined by Lemma 8 . Using Lemma 8 and under the assumption $q^{1-\frac{n}{m}} \sqrt{\frac{m}{2 \pi e}}<q$, we require a gap of size approximately

$$
\frac{\lambda_{2}}{\lambda_{1}}=\frac{q^{1-\frac{n}{m}} \sqrt{\frac{1}{2 e}}}{\epsilon^{\prime} \alpha q}
$$

and so we require a $\delta_{0}$ determined by $q^{1-\frac{n}{m}} \sqrt{\frac{1}{2 e}} \geq \tau \delta_{0}^{m} \epsilon^{\prime} \alpha q$. The determination of $\tau$ is discussed at length in [AFG14] but we have that $\tau \leq 0.4$ (depending on the algorithm) for a success

\footnotetext{
${ }^{3}$ To avoid notational conflict we refer to their constant $c>1$ as a constant $\epsilon^{\prime}>1$ and we replace their $s$ for the width parameter of the Gaussian with our $\alpha q$.
} 
probability of 0.1 based on the experimental results in [AFG14,GN08]. We stress that no data is publicly available on $\tau$ for smaller success probabilities. In [AFG14] it is shown that for a fixed $\delta_{0}$ the optimal subdimension is $m=\sqrt{\frac{n \log q}{\log \delta_{0}}}$ as in Section 3.3. We may use this to determine $\delta_{0}$ using the expression above (where for simplicity we assume equality).

Lemma 9. Given an LWE instance characterised by $n, \alpha, q$. Any lattice reduction algorithm achieving log root-Hermite factor

$$
\log \delta_{0}=\frac{\log ^{2}\left(\epsilon^{\prime} \tau \alpha \sqrt{2 e}\right)}{4 n \log q}
$$

solves LWE with success probability greater than

$$
\epsilon_{\tau} \cdot\left(1-\left(\epsilon^{\prime} \cdot \exp \left(\frac{1-\epsilon^{\prime 2}}{2}\right)\right)^{m}\right)
$$

for some $\epsilon^{\prime}>1$ and some fixed $\tau \leq 1$, and $0<\epsilon_{\tau}<1$ as a function of $\tau$.

Proof. From the above discussion, and assuming for simplicity an equality, we require a $\delta_{0}$ determined by the following equation:

$$
q\left(1-\frac{n}{\sqrt{\frac{n \log q}{\log \delta}}}\right) \sqrt{\frac{1}{2 e}}=\epsilon^{\prime} \tau \alpha q \delta_{0}^{\sqrt{\frac{n \log q}{\log \delta_{0}}}}
$$

Rearranging, we obtain

$$
\begin{aligned}
\left(1-\frac{n}{\sqrt{\frac{n \log q}{\log \delta_{0}}}}\right) \log q+\log \sqrt{\frac{1}{2 e}} & =\log \left(\epsilon^{\prime} \tau \alpha q\right)+\sqrt{\frac{n \log q}{\log \delta_{0}}} \log \delta_{0} \\
\left(\frac{\sqrt{\frac{n \log q}{\log \delta_{0}}}-n}{\sqrt{\frac{n \log q}{\log \delta_{0}}}}\right) \log q-\sqrt{\frac{n \log q}{\log \delta_{0}}} \log \delta_{0} & =\log \left(\epsilon^{\prime} \tau \alpha q\right)-\log \sqrt{\frac{1}{2 e}} \\
\left(\frac{\sqrt{\frac{n \log q}{\log \delta_{0}}}-n}{\sqrt{\frac{n \log q}{\log \delta_{0}}}}\right) \log q-\sqrt{\frac{n \log q}{\log \delta_{0}}} \log \delta_{0} & =\log \left(\epsilon^{\prime} \tau \alpha q \sqrt{2 e}\right) \\
\sqrt{\frac{n \log q}{\log \delta_{0}}} \log \left(\epsilon^{\prime} \tau \alpha \sqrt{2 e}\right) & =2 n \log q \\
\frac{n \log q}{\log \delta_{0}} \log ^{2}\left(\epsilon^{\prime} \tau \alpha \sqrt{2 e}\right) & =4 n^{2}(\log q)^{2} \\
\frac{\log ^{2}\left(\epsilon^{\prime} \tau \alpha \sqrt{2 e}\right)}{4 n \log q} & =\log \delta_{0} .
\end{aligned}
$$

Finally, the success probability is computed as the probability the gap is as required in Lemma 8 multiplied by the success probability of our algorithm $\epsilon_{\tau}$. 
Corollary 7. Given an LWE instance characterised by $n, q=n^{c}, \alpha q=\sqrt{n}$. Any lattice reduction algorithm achieving log root-Hermite factor

$$
\log \delta_{0}=\frac{\left((c-1 / 2) \log n-\log \left(\epsilon^{\prime} \tau \sqrt{2 e}\right)\right)^{2}}{4 c n \log n}
$$

solves LWE with success probability greater than

$$
\epsilon_{\tau} \cdot\left(1-\left(\epsilon^{\prime} \cdot \exp \left(\frac{1-\epsilon^{\prime 2}}{2}\right)\right)^{m}\right)
$$

for some $\epsilon^{\prime} \approx 1$ and some fixed $\tau \leq 1$ and $0<\epsilon_{\tau}<1$ as a function of $\tau$.

Comparing Corollary 7 with Corollary 6 we find that solving LWE via BDD by reducing to uSVP is more efficient than solving LWE via one call to an algorithm solving SIS whenever $\log (1 /(\tau \sqrt{2 e}))>\log (f(\epsilon))$ under the condition that $\epsilon \approx \epsilon_{\tau}$ so that the success probabilities are equal in both cases.

However, using Kannan's embedding is not necessarily more efficient than the decoding approach discussed in Section 5.4, as the following example highlights.

Example 4. Letting $n=256, q=65537$ and $\sigma=\alpha q / \sqrt{2 \pi} \approx 25.53$ following [Reg09] and choosing $t=1, \tau^{\prime}=0.310$ and $\epsilon^{\prime}=1.01$ we get a target $\delta_{0}=1.004634$ by Lemma 9 and thus $m=784$. According to [AFG14] we have $\epsilon_{\tau}=0.1$. Our estimator predicts block size $k=321$ and $2^{205}$ clock cycles for performing this lattice reduction. In contrast, performing the decoding approach from Section 5.4 is predicted to cost $2^{172}$ clock cycles overall to perform lattice reduction which achieves root-Hermite factor $\delta_{0}=1.005198$ and to run the final decoding stage.

Finally, suppose $t<\|\mathbf{e}\|$. In this case no efficient method for determining $\frac{\lambda_{2}}{\lambda_{1}}$ is known. The assumption in [AFG14] which attempts to overcome this is that the same size of gap is required as it is in the case that $t=\|\mathbf{e}\|$. A modified value for $\tau$, denoted $\tau^{\prime}$ is introduced which relates to the gap from the case $t=e$ when actually computing with $t=1$. Setting $t=1$ is typically more efficient than $t=\|\mathbf{e}\|$ and we have $\tau^{\prime} \approx 0.3$, see [AFG14] for details.

\subsection{Arora-Ge and Gröbner Bases}

Arora and Ge proposed an alternative approach to solving Search-LWE by setting up a system of noise-free non-linear polynomials of which the secret $\mathbf{s}$ is a root [AG11]. This approach solves for s directly.

In particular, [AG11] offers an algorithm for solving Search-LWE in time $2^{\tilde{\mathcal{O}}\left(n^{2 \xi}\right)}$, where $\xi$ is a constant such that $\alpha q=n^{\xi}$. The algorithm proceeds by assuming that the error always falls in the range $[-t, t]$ for some $t \in \mathbb{Z}$ such that $d=2 t+1<q$. This follows from the chance of falling outside this interval dropping exponentially fast (cf. Lemma 4).

Polynomials are constructed from the observation that the error, when falling in this range, is always a root of the polynomial $P(x)=x \prod_{i=1}^{t}(x+i)(x-i)$. Then, we know the secret $\mathbf{s}$ is a root of $P(\mathbf{a} \cdot \mathbf{x}-c)$ constructed from LWE samples. In the Arora-Ge algorithm the system of nonlinear equations constructed this way is solved by replacing each monomial with a new variable and solving the resulting linear system. However, this means that we need $\mathcal{O}\left(n^{2 t+1}\right)$ samples. As we increase the number of samples, we increase the probability that the error falls outside of the interval $[-t, t]$. We then have to increase the range, leading to a larger degree, which requires even more samples. Balancing these two requirements of keeping the degree low and acquiring enough samples, the overall complexity is given by the following result. 
Theorem 5 (Theorem 5 in [ACFP14]). Let $n, q, \sigma=\alpha q$ be parameters of an LWE instance, and as before let $\omega$ denote the linear algebra constant. Let $D_{\mathrm{AG}}=8 \sigma^{2} \log n+1$. If $D_{\mathrm{AG}} \in$ o $(n)$ then the Arora-Ge algorithm solves Search-LWE in time complexity

$$
\mathcal{O}\left(2^{\omega \cdot D_{\mathrm{AG}} \log \frac{n}{D_{\mathrm{AG}}}} \cdot \sigma q \log q\right)=\mathcal{O}\left(2^{8 \omega \sigma^{2} \log n\left(\log n-\log \left(8 \sigma^{2} \log n\right)\right)} \cdot \operatorname{poly}(n)\right)
$$

and memory complexity

$$
\mathcal{O}\left(2^{2 \cdot D_{\mathrm{AG}} \log \frac{n}{D_{\mathrm{AG}}}} \cdot \sigma q \log q\right)=\mathcal{O}\left(2^{16 \sigma^{2} \log n\left(\log n-\log \left(8 \sigma^{2} \log n\right)\right)} \cdot \operatorname{poly}(n)\right) .
$$

If $n \in o\left(D_{\mathrm{AG}}\right)$ then the Arora-Ge algorithm solves Search-LWE in time complexity

$$
\mathcal{O}\left(2^{\omega n \log \frac{D_{\mathrm{AG}}}{n}} \cdot \sigma q \log q\right)=\mathcal{O}\left(2^{\omega n \log \left(8 \sigma^{2} \log n\right)-\omega n \log n} \cdot \operatorname{poly}(n)\right)
$$

and memory complexity

$$
\mathcal{O}\left(2^{2 n \log \frac{D_{\mathrm{AG}}}{n}} \cdot \sigma q \log q\right)=\mathcal{O}\left(2^{2 n \log \left(8 \sigma^{2} \log n\right)-2 n \log n} \cdot \operatorname{poly}(n)\right) .
$$

Remark 7. Specialising to $\sigma=\sqrt{n}$ the complexity is $\mathcal{O}\left(2^{(2+\epsilon) \omega n \log \log n}\right)$. In this case, the AroraGe algorithm is asymptotically slower than the BKW algorithm and lattice reduction if sieving is used to implement the SVP oracle, but asymptotically faster than lattice reduction if enumeration is used to implement the SVP oracle.

This can be improved by using Gröbner basis techniques [ACFP14]. In particular, to solve via linearisation as in [AG11], we require $\mathcal{O}\left(n^{d}\right)$ equations, but Gröbner basis algorithms will work when fewer equations than this are available at the cost of a more expensive solving step. In particular, the complexity of computing a Gröbner basis is $\mathcal{O}\left(m D_{\text {reg }}\left(\begin{array}{c}n+D_{\text {reg }} \\ D_{\text {reg }}\end{array}\right)\right.$, where $D_{\text {reg }}$ is the degree of regularity of the ideal $\mathcal{I}$ spanned by the polynomials. The degree $D_{\text {reg }}$ is the index of the first non-positive coefficient of the Hilbert series expansion of the ideal $\mathcal{I}$. In general, it is hard to compute the Hilbert series, but for semi-regular sequences, it has an easy form. A semi-regular sequence of $m$ polynomials of degree $d$ in $n$ variables is a sequences with the following Hilbert series:

$$
H_{\mathcal{I}}(z):=\frac{\left(1-z^{d}\right)^{m}}{(1-z)^{n}}
$$

It is assumed that random systems behave like semi-regular sequences. A justification as to why this is a reasonable assumption is given in [ACFP14]. Thus, assuming our non-linear equations behave like random equations of the same degree, we can estimate the cost of solving LWE by expanding this power series until the first non-positive coefficient. In particular, assuming $\alpha q=\sqrt{n}$ we get:

Theorem 6 ([ACFP14]). Let $\left(\mathbf{a}_{i}, b_{i}\right)$ for $i \geq 1$ be elements of $\mathbb{Z}_{q}^{n} \times \mathbb{Z}_{q}$ sampled according to $L_{\mathbf{s}, \chi}$ with $\alpha q=\sqrt{n}$. There is an algorithm recovering the secret with time complexity $\mathcal{O}\left(2^{2.35 \omega n+1.13 n}\right)$, memory complexity $\mathcal{O}\left(2^{5.85 n}\right)$ and sample complexity $m=\exp \left(\frac{\pi}{4} \cdot n\right)$.

Hence, for $\alpha q=\sqrt{n}$ applying Gröbner basis algorithms is in the same complexity class as the BKW algorithm or lattice reduction when sieving implements the SVP oracle, albeit with a larger leading constant in the exponent.

Remark 8. The complexity depends on $\alpha q$, which corresponds to the degree, and $n$, which corresponds to the number of variables. Adjusting $q$ while keeping $\alpha q$ the same will not affect the runtime. 


\section{Small Secret Variants}

In several applications based on LWE, the secret $\mathbf{s}$ is not chosen uniformly at random from $\mathbb{Z}_{q}$ but instead chosen from a different distribution where all the components $\mathbf{s}_{(i)}$ are "small", e.g. they are chosen from $\{0,1\}$ or $\{-1,0,1\}$. In this section we consider the complexity of solving LWE in this special case. We characterise an instance by $n, \alpha, q, \psi$ where $\psi$ is the distribution of $\mathbf{s}_{(i)}$.

We note that there is a gap between security reductions and the best known algorithms for solving LWE with binary secrets. On the one hand, theoretical results show that for an LWE instance with a binary secret to be as hard as general LWE in dimension $n$ a dimension of $n \log q$ is sufficient $\left[\mathrm{BLP}^{+} 13, \mathrm{MP} 13\right]$. On the other hand, the best known algorithms for solving LWE with a binary secret from [BG14] manages to solve LWE instances with a binary secret and in dimension $n \log \log n$ in about the same complexity as it would take to solve a standard LWE instance in dimension $n$. Hence, based on the currently best known attacks we would conclude that we only need to increase the dimension to $n \log \log n$ instead of $n \log q$. Hence, there is room for improvement either for algorithms or for security reductions.

\subsection{Exhaustive Search}

In Section 5.1 above we saw that exhaustive search can be solved by checking all the vectors within a sphere of radius $t \alpha q$, for some small parameter $t=\omega(\sqrt{\log n})$, which is essentially the size of the secret. Even without explicitly knowing $\psi$, we can restrict our search to the support of $\psi$, for example $\{-1,0,1\}$. We can simply check all possible $\mathbf{s}$ with $\mathbf{s}_{(i)}$ chosen from this set. Then by the same argument as in Theorem 1 , exhaustive search will take time $m \cdot 3^{n} \cdot(2 n)=2^{n \log 3+\log n+1+\log m}$ if $\mathbf{s}_{(i)} \in\{-1,0,1\}$.

As observed in [BG14] we can also combine exhaustive search with other algorithms to improve the complexity by guessing, say, $g$ components of the secret and then running our algorithm on the reduced small secret LWE instance of dimension $n-g$. With this strategy any algorithm discussed below can be turned into an algorithm which has at most the cost of exhaustive search.

MITM By exactly the same argument as in Theorem 2, whatever time we would expect it to take to solve exhaustive search (which depends on $\psi$ ), we may achieve essentially the same speed up as we would do applying a Meet-in-the-Middle strategy to a general LWE instance. So, if the components $\mathbf{s}_{(i)}$ are selected from $\{-1,0,1\}$ then an MITM strategy will take time $\mathcal{O}\left(3^{n / 2}\right)$ and require $\operatorname{poly}(n) \cdot 3^{n / 2}$ memory.

\subsection{Modulus Switching for Lattice Reduction}

For an LWE instance parametrised by $n, \alpha, q$ and with a small secret, we may apply modulus switching and consider the instance $\bmod p$ where $p<q$. This allows for a larger $\delta_{0}$ than would be required for an instance parametrised by the same $n, \alpha, q$ and with a secret where $\mathbf{s}_{(i)}$ is chosen at random from $\mathbb{Z}_{q}$. After modulus switching, the transformed instance has an error which is slightly larger and its distribution is no longer exactly a discrete Gaussian. Nonetheless, heuristically, algorithms which solve LWE still solve these LWE-like problem instances and so we assume that after modulus switching, we have an LWE instance characterised by $n, \sqrt{2} \alpha$ and $p$. So, when we have a small secret we may obtain a speed up by modulus switching before performing lattice reduction (for example, as described in Sections 5.3, 5.4 and 5.5). 
As an example we consider distinguishing LWE by lattice reduction as in Section 5.3. As with a general secret, we assume the size of the small vector we aim to output is $\|\mathbf{v}\|=\frac{1}{\sqrt{2} \alpha} f(\epsilon)$.

Lemma 10. Let a small secret LWE instance be characterised by $n, \alpha, q$ and $\mathbf{s}_{(i)} \leftarrow_{\$} \psi$. Then the $\log$ root-Hermite factor $\log \delta_{0}$ required to distinguish by lattice reduction is

$$
\log \delta_{0}=\frac{\left(\log \left(\sqrt{2} \alpha \frac{1}{f(\epsilon)}\right)\right)^{2}}{4 n \log p}
$$

for $p$ such that

$$
\left\|\left\langle\frac{p}{q} \cdot \mathbf{a}-\left\lfloor\frac{p}{q} \cdot \mathbf{a}\right\rceil, \mathbf{s}\right\rangle\right\| \approx \frac{p}{q} \cdot\|e\|
$$

Proof. Using Lemma 2, modulus switch and transform the LWE instance (a, $c) \in \mathbb{Z}_{q}^{n} \times \mathbb{Z}_{q}$ into an LWE instance in $\mathbb{Z}_{p}^{n} \times \mathbb{Z}_{p}$. Now the instance is parametrised by $n, p, \sqrt{2} \alpha$ and by the same argument as in Lemma 7 we require $\delta_{0}=2 \frac{\left(\log \left(\sqrt{2} \alpha \frac{1}{f(\epsilon)}\right)\right)^{2}}{4 n \log p}$.

Corollary 8. Let a small secret LWE instance be characterised by $n, \alpha, q$ and $\psi$, suppose $\alpha q=\sqrt{n}$ and $q=n^{c}$ and $\psi$ is such that the standard deviation of the elements in the secret $\mathbf{s}$ is $\sigma_{s}$. Then the log root-Hermite factor $\log \delta_{0}$ that is required is

$$
\log \delta_{0}=\frac{\left(\log \left(\frac{\sqrt{2}}{f(\epsilon)} n^{\frac{1}{2}-c}\right)\right)^{2}}{4 n\left(\log \left(\frac{\sqrt{\pi}}{\sqrt{6}} \sigma_{s}\right)+c \log n\right)}
$$

Proof. From Lemma 2 we have $p=\frac{\sigma_{s}}{\alpha} \sqrt{\frac{2 \pi n}{12}}=\frac{\sigma_{s} \sqrt{2} \sqrt{\pi} \sqrt{n}}{\sqrt{12} \alpha}=\frac{\sqrt{\pi}}{\sqrt{6}} \sigma_{s} n^{c}$.

By Lemma 10 we have

$$
\begin{aligned}
\log \delta_{0} & =\frac{\left(\log \left(\sqrt{2} \alpha \frac{1}{f(\epsilon)}\right)\right)^{2}}{4 n \log p}=\frac{\left(\log \left(\sqrt{2} \alpha \frac{1}{f(\epsilon)}\right)\right)^{2}}{4 n \log \left(\frac{\sqrt{\pi}}{\sqrt{6}} \sigma_{s} n^{c}\right)} \\
& =\frac{\left(\log \left(\frac{\sqrt{2}}{f(\epsilon)} n^{\frac{1}{2}-c}\right)\right)^{2}}{4 n\left(\log \left(\frac{\sqrt{\pi}}{\sqrt{6}} \sigma_{s}\right)+c \log n\right)}
\end{aligned}
$$

Example 5. Setting $n=256, q=65537$ and $\sigma=\alpha q / \sqrt{2 \pi} \approx 25.53$ following [Reg09] and $\psi=$ $\mathcal{U}\left(\mathbb{Z}_{2}\right)$ we have $\sigma_{s}=0.5$. Modulus switching reduces the size of the modulus to $p=5928$. Picking $\epsilon=0.1$ we get a target $\delta_{0}=1.0046578$ by Lemma 7 and thus $m=781$. Computing the expected number of clock cycles according to the various models available to us, we end up with the following estimates:

\begin{tabular}{rrr}
\hline model & block size $k$ & log clock cycles \\
\hline enum & 318 & 209 \\
sieve & 318 & 154 \\
\hline
\end{tabular}




\subsection{Bai and Galbraith's Embedding}

Bai and Galbraith [BG14] show that for binary secret (that is, $\mathbf{s}_{(i)} \leftarrow\{0,1\}$ or $\mathbf{s}_{(i)} \leftarrow\{-1,0,1\}$ ) we may embed our LWE lattice into a different lattice with uSVP structure than the one considered in Section 5.5. Let $m^{\prime}=m+n$. The lattice is then $L=\left\{\mathbf{v} \in \mathbb{Z}^{m^{\prime}} \mid \mathbf{A}^{\prime} \mathbf{v} \equiv 0 \bmod q\right\}$ where $\mathbf{A}^{\prime}=\left(\mathbf{A} \mid \mathbf{I}_{m}\right)$. This method is more efficient than the usual Kannan embedding lattice as discussed in Section 5.5.

The target short vector is now (s\|e) (as opposed to e as in the Kannan embedding case) which contains among its components those of s. Bai and Galbraith observe that this enables us to take advantage of the smallness of $\mathbf{s}$ [BG14]. In particular, it is its smallness compared with the size of the error which is exploited. That is, where we have $\|\mathbf{s}\| \ll\|\mathbf{e}\|$, we may rescale the lattice into which the instance is embedded, increasing its volume. This increases the $\delta_{0}$ which is required to solve the instance. In the case $\mathbf{s}_{(i)} \leftarrow\{-1,0,1\}$, after an appropriate rescaling, the volume of the lattice is increased by $\sigma^{n}$, where $\sigma=\frac{\alpha q}{\sqrt{2 \pi}}$ is approximately the standard deviation of the error. In the case $\mathbf{s}_{(i)} \leftarrow\{0,1\}$ the volume is increased by $(2 \sigma)^{n}$ because we can scale by $2 \sigma$ and then rebalance. We note that (in the terminology of Section 5.5) Bai and Galbraith use $t=1$ rather than $t=\|\mathbf{e}\|$. In our lemma below, we adapted their theorem to $t=\|\mathbf{e}\|$. In our experiments we then use $\tau^{\prime}$ to estimate the cost of lattice reduction for $t=1$.

Lemma 11. Let a small secret LWE instance be characterised by $n, \alpha, q$, let $\mathbf{s}_{(i)} \leftarrow_{\$}\{a, \ldots, b\}$, let $\xi=2 /(b-a)$ and let $\sigma=\frac{\alpha q}{\sqrt{2 \pi}}$. Any lattice reduction algorithm achieving log root-Hermite factor:

$$
\log \delta=\frac{(\log (q / \sigma)-\log (2 \tau \sqrt{\pi e}))^{2} \cdot \log (q / \sigma)}{n(2 \log (q / \sigma)-\log \xi)^{2}}
$$

solves LWE by reducing BDD to uSVP for some fixed $\tau \leq 1$ if we have that $\left(q^{m}(\xi \sigma)^{n}\right)^{1 /(m+n)} \sqrt{\frac{m+n}{2 \pi e}} \leq$ $q$ where $m=m^{\prime}-n=\sqrt{\frac{n(\log q-\log \sigma)}{\log \delta}}-n$.

Proof. We observe that scaling and rebalancing for a secret sampled from the interval $[a, \ldots, b]$ increases the volume by a factor of $(\xi \sigma)^{n}$. Hence, by [BG14, Section 6.2] and the assumption that $\left(q^{m}(\xi \sigma)^{n}\right)^{1 /(m+n)} \sqrt{\frac{m+n}{2 \pi e}} \leq q$ we have a gap of roughly $\frac{\lambda_{2}}{\lambda_{1}}=\frac{\left(q^{m}(\xi \sigma)^{n}\right)^{1 /(m+n)} \sqrt{\frac{m+n}{2 \pi e}}}{\sqrt{2 m+n} \cdot \sigma}$.

Following the notation of [BG14], let $m^{\prime}=m+n$. By the same argument as the discussion in Section 5.5 we have $\frac{\lambda_{2}}{\lambda_{1}} \geq \tau \delta^{m^{\prime}}$. Again for simplicity we assume equality. By [BG14, Lemma 1] the optimal value of $m^{\prime}$ is $m^{\prime}=\sqrt{\frac{n(\log q-\log \sigma)}{\log \delta}}$. Therefore we have:

$$
\frac{\left(q^{m^{\prime}-n}(\xi \sigma)^{n}\right)^{\left(\frac{1}{m^{\prime}}\right)}}{\sqrt{4 \pi e} \sigma}=\tau \delta^{m^{\prime}}
$$

Taking logarithms and rearranging we get:

$$
\left(1-\frac{n}{m^{\prime}}\right) \log q+\frac{n}{m^{\prime}} \log (\xi \sigma)=m^{\prime} \log \delta+\log \sigma+\log (\tau \sqrt{4 \pi e})
$$

Solving for $\log \delta_{0}$ :

$$
\log \delta_{0}=\frac{m^{\prime}(\log (q / \sigma)-\log (\tau \sqrt{4 \pi e}))+n \log (\xi)-n \log (q / \sigma)}{m^{\prime 2}}
$$


Substituting $m^{\prime}=\sqrt{\frac{n(\log q-\log \sigma)}{\log \delta}}$ :

$$
\begin{aligned}
& \log \delta_{0}=\frac{\sqrt{\frac{n(\log q-\log \sigma)}{\log \delta}}(\log (q / \sigma)-\log (\tau \sqrt{4 \pi e}))+n \log (\xi)-n \log (q / \sigma)}{\sqrt{\frac{n(\log q-\log \sigma)}{\log \delta}}^{2}} \\
& 1=\frac{\sqrt{\frac{n(\log q-\log \sigma)}{\log \delta}}(\log (q / \sigma)-\log (\tau \sqrt{4 \pi e}))+n \log (\xi)-n \log (q / \sigma)}{n \log (q / \sigma)}
\end{aligned}
$$

Solving for $\sqrt{\frac{n(\log q-\log \sigma)}{\log \delta}}$ :

$$
\sqrt{\frac{n(\log q-\log \sigma)}{\log \delta}}=\frac{2 n \log (q / \sigma)-n \log (\xi)}{\log (q / \sigma)-\log (\tau \sqrt{4 \pi e})}
$$

Finally, solving for $\log \delta_{0}$ :

$$
\log \delta=\frac{(\log (q / \sigma)-\log (\tau \sqrt{4 \pi e}))^{2} \cdot \log (q / \sigma)}{n(2 \log (q / \sigma)-\log \xi)^{2}}
$$

Remark 9. Specialising Lemma 11 to $\mathbf{s}_{(i)} \leftarrow_{\$}\{-1,0,1\}$ and hence $\xi=1$ gives

$$
\log \delta=\frac{(\log \alpha \tau \sqrt{2 e})^{2}}{4 n\left(\log q-\log \frac{\alpha q}{\sqrt{2 \pi}}\right)} .
$$

Bai and Galbraith also observe that, perhaps counterintuitively, modulus switching does not improve their algorithm. This is because modulus switching results in a smaller rescaling factor and hence leaves a smaller gap.

\subsection{Small Secret BKW}

In this section we consider the small secret variant of BKW described in [AFFP14]. In this work $\psi$ is not specified but it is assumed that the $\mathbf{s}_{(i)}$ are chosen from $\{-1,0,1\}$ or $\{0,1\}$. The authors employ their own variant of BKW to achieve a complexity reduction for solving BKW with small secret. Their technique is lazy modulus switching, a variant of modulus switching. To maximise complexity improvements, the authors only modulus switch when necessary, and employ techniques such as searching for collisions $\bmod p$ but remaining in $\mathbb{Z}_{q}$ when doing arithmetic on the rows.

Theorem 7 ([AFFP14]). Let $n \geq 1$ be the dimension of the LWE secret vector, $q$ be a modulus, $b \in \mathbb{Z}$ with $1 \leq b \leq n$ and $\sigma_{s}$ the standard deviation of the secret vector components. Let also $\sigma_{r}$ be the variance of random elements in $\mathbb{Z}_{r}$. Define $a=\lceil n / b\rceil$ and pick a pair $\left(p, m^{*}\right)$ such that $b \sigma_{r}^{2} \sigma_{s}^{2} \sum_{i=0}^{a-1} \mathbf{v}_{(i)} \leq 2^{a} \sigma$. Then $B_{\mathbf{s}, \chi}(b, a-1, p)$ will return $\left(\tilde{\mathbf{a}}_{0}, \tilde{c}_{0}\right), \ldots,\left(\tilde{\mathbf{a}}_{m-1}, \tilde{c}_{m-1}\right)$ where $\tilde{c}_{i}$ has standard deviation $\leq \sqrt{2^{a+1}} \sigma$. Furthermore this costs $\frac{p^{b}}{2}\left(\frac{a(a-1)}{2}(n+1)\right)+\left(m+m^{*}\right)$ na additions in $\mathbb{Z}_{q}$ and $\frac{a p^{b}}{2}+m+m^{*}$ calls to $L_{\mathbf{s}, \chi}$.

In particular, for a typical choice of parameters: $q \approx n^{c}$ for some small $c \geq 1, a=\log n, b=\frac{n}{\log n}$, recall that standard BKW has complexity $\mathcal{O}\left(2^{c n} \cdot n \log ^{2} n\right)$. Here the complexity of solving is 
$\mathcal{O}\left(2^{n\left(c+\frac{\log d}{\log n}\right)} \cdot n \log ^{2} n\right)$, using naive modulus switching. Using lazy modulus switching (Corollary 3 of [AFFP14]), the complexity of solving is $\mathcal{O}\left(2^{n\left(c+\frac{\log d-\frac{1}{2} \log \log n}{\log n}\right)} \cdot n \log ^{2} n\right)$, where in both cases $0<d \leq 1$ is a constant.

\subsection{Arora-Ge and Gröbner Bases}

We may exploit small secrets when reducing LWE to solving a non-linear system of equations as in Section 5.6. To encode that our secret is small, we add low-degree equations of the form $\prod_{i=0}^{s-1} x-j_{i}$ where $s$ is the cardinality of the support for $\psi$ and $j_{i}$ are the elements of the support. We may then expand the Hilbert series to establish the expected degree of semi-regularity.

\section{Examples}

In this section we use our estimator to estimate the cost of running the algorithms discussed in Sections 5 and 6 for parameter sets from the literature. Our estimator is available at https: //bitbucket.org/malb/lwe-estimator. We consider the following parameter sets.

\section{Regev}

These are Regev's example choices for parameters from [Reg09]. We use [AFC $\left.{ }^{+} 13\right]$ to pick $q \approx n^{2}$ and $\alpha=1 /\left(\sqrt{2 \pi n} \log _{2}^{2} n\right)$.

\section{LindnerPeikert}

\section{FHE}

We use $\left[\mathrm{AFC}^{+} 13\right]$ to select parameters as suggested in [LP11] given $n$.

Given $n$ and the multiplicative depth $L$ we set $q=2^{16.5 \cdot L+5.4} \cdot 8^{2 L-3} \cdot n^{L}$ and $\alpha=3.2 \sqrt{2 \pi} / q$ inspired by parameters suggested in [GHS12c]. We always assume $\mathbf{s}_{(i)} \leftarrow \$\{0,1\}$, which means our secret is a bit bigger than in [GHS12c] where the secret has Hamming weight $\lambda / 2$ regardless of dimension.

In our tables "MitM" refers to the Meet-in-the-Middle algorithm given in Section 5.1, "BKW" to the BKW algorithm discussed in Section 5.2, "SIS" to the algorithm discussed in Section 5.3, "DEC" to the algorithm discussed in Section 5.4, "Kannan" to the algorithm discussed in Section 5.5, "Bai-Gal" to the algorithm discussed in Section 6.3 and "Arora-GB" to applying Gröbner basis algorithms as discussed in Section 5.6. In those tables concerning small secret variants, the same labels refer to the small secret variants of the respective algorithms.

The columns "bop" refer to estimated bit operations which we identify with CPU clock cycles. This identification slightly favours lattice reduction algorithms compared to other algorithms, because CPUs do more than one operation per bit per clock cycle. The columns "mem" refer to storage requirements of elements in $\mathbb{Z}_{q}$. The columns " $L_{\mathbf{s}, \chi}$ " refers to the number of calls to the LWE oracle. The columns "bkz2" resp. "sieve" refer to BKZ 2.0 estimates based on the row "enum" resp. "sieve" in Table 1. We use the "bkz2" estimates to optimise parameters for latticebased algorithms. The column "enum" gives the number of enumerations in the decoding stage of "DEC". The column "g" is the number of components that are guessed before running the respective algorithms as discussed in Section 6.1 (this only applies to small secret instances). All columns list the logarithm to base two of their respective values.

In all cases, costs are overall, i.e. we give an estimate for the overall cost of solving, including repeated trials and repeated guesses. If "-" is given instead of a number, it means our estimator 
did not return a value or was not run because it does not cover this particular case. This can happen when estimates only exist for special cases such as when applying Gröbner bases. We always use $\tau^{\prime}=0.3$ when considering Kannan embedding or the embedding by Bai and Galbraith. For each choice of parameter set and for each $n$, we highlight the entry giving the runtime of the algorithm which runs fastest in that case.

\begin{tabular}{c|c|c|c|c|c|c|c|c|c|c|c|c|c}
\hline & \multicolumn{3}{c}{ BKW } & \multicolumn{3}{c}{ SIS } & \multicolumn{3}{c}{ DEC } & \multicolumn{2}{c}{ Kannan } \\
\hline$n$ & bop & mem & $L_{\mathbf{s}, \chi}$ & bkz2 & sieve & $L_{\mathbf{s}, \chi}$ & bop & enum & $L_{\mathbf{s}, \chi}$ & bkz2 & sieve & $L_{\mathbf{s}, \chi}$ \\
\hline 64 & 57 & 50 & 45 & $\mathbf{3 4}$ & 50 & 12 & $\mathbf{3 4}$ & 18 & 10 & 35 & 50 & 13 \\
128 & 101 & 93 & 87 & 75 & 86 & 27 & 65 & 49 & 11 & $\mathbf{6 3}$ & 73 & 14 \\
256 & 189 & 181 & 174 & 205 & 171 & 52 & 168 & 152 & 45 & 207 & $\mathbf{1 5 3}$ & 15 \\
512 & 366 & 358 & 350 & 566 & 339 & 81 & 453 & 437 & 46 & 665 & $\mathbf{3 3 5}$ & 16 \\
1024 & $\mathbf{7 2 1}$ & 712 & 703 & 1478 & 732 & 206 & 1203 & 1187 & 111 & 1962 & 747 & 17 \\
\hline
\end{tabular}

Table 3. Regev

\begin{tabular}{c|c|c|c|c|c|c|c|c|c|c|c|c|c|c|c|c|c|c}
\hline & \multicolumn{3}{c}{ BKW } & \multicolumn{2}{c}{ SIS } & \multicolumn{2}{c}{ DEC } & \multicolumn{2}{c}{ Kannan } & \multicolumn{2}{c}{ Arora-GB } \\
\hline$n$ & bop & mem & $L_{\mathbf{s}, \chi}$ & bkz2 & sieve & $L_{\mathbf{s}, \chi}$ & bop & enum & $L_{\mathbf{s}, \chi}$ & bkz2 & sieve & $L_{\mathbf{s}, \chi}$ & bop & mem & $L_{\mathbf{s}, \chi}$ \\
\hline 64 & 54 & 47 & 42 & $\mathbf{3 4}$ & 49 & 12 & $\mathbf{3 4}$ & 18 & 10 & 35 & 50 & 13 & 289 & 286 & 92 \\
128 & 89 & 82 & 76 & 70 & 81 & 22 & 60 & 44 & 11 & $\mathbf{5 7}$ & 69 & 14 & 505 & 501 & 389 \\
256 & 160 & 151 & 146 & 179 & 156 & 47 & 146 & 130 & 44 & 181 & $\mathbf{1 4 0}$ & 15 & - & - & - \\
512 & $\mathbf{2 8 9}$ & 281 & 273 & 476 & 308 & 86 & 376 & 361 & 45 & 579 & 303 & 16 & - & - & - \\
1024 & $\mathbf{5 4 8}$ & 541 & 532 & 1190 & 636 & 201 & 954 & 938 & 110 & 1643 & 652 & 17 & - & - & - \\
\hline
\end{tabular}

Table 4. LindnerPeikert

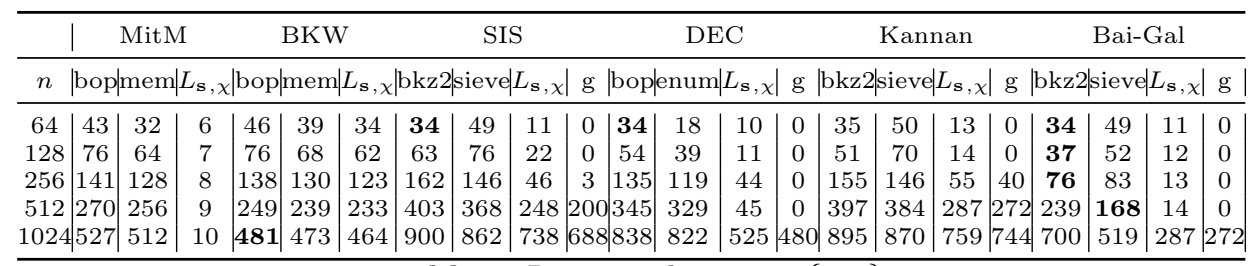

Table 5. Regev with $\mathbf{s}_{(i)} \leftarrow \$\{0,1\}$

\section{Discussion}

The problems of giving the concrete hardness of the Learning with Errors problem are manifold.

No closed formulas. For most algorithms, there is no sufficiently precise closed formula which expresses the running time in terms of the parameters specifying the problem (e.g. $n, q, \alpha$ ), mainly due to a lack of a closed formula for lattice reduction as a function of $\delta_{0}$. This makes direct comparisons difficult. This problem is addressed by the Sage module, enabling us to estimate running times of the various algorithms for particular parameter choices.

The results of applying this Sage module broadly agree with the literature. For example, by our estimates the parameter choices made in [GHS12c] are too conservative, as first observed by van de Pol and Smart [vdPS13], even in light of specialised algorithms exploiting the presence of a 


\begin{tabular}{|c|c|c|c|c|c|c|c|c|c|c|c|c|c|c|c|c|c|c|c|c|c|}
\hline & \multicolumn{3}{|c|}{ MitM } & \multicolumn{3}{|c|}{ BKW } & \multicolumn{3}{|c|}{ SIS } & \multicolumn{3}{|c|}{ DEC } & \multicolumn{4}{|c|}{ Kannan } & \multicolumn{5}{|c|}{ Bai-Gal } \\
\hline$n$ & & & & & & & & & & & & & & & & & & & & & $\mathrm{~g}$ \\
\hline 64 & 42 & 32 & 6 & 46 & 39 & 34 & 34 & 49 & 11 & 34 & 18 & 10 & 0 & 35 & 50 & 13 & 0 & 34 & 49 & 11 & 0 \\
\hline 128 & 75 & 64 & 7 & 75 & 68 & 62 & 75 & 85 & 26 & \begin{tabular}{l|l|}
0 & 60
\end{tabular} & 44 & 19 & 0 & 62 & 73 & 14 & 0 & 37 & 52 & 12 & 0 \\
\hline 256 & 141 & 128 & 8 & 136 & 129 & 122 & 191 & 185 & 97 & $60|157|$ & 141 & 44 & 0 & 181 & 184 & 110 & 96 & 90 & 91 & 13 & 0 \\
\hline 512 & 270 & 256 & 9 & 255 & 248 & 240 & 450 & 449 & 369 & $336 \mid 413$ & 397 & 237 & 192 & 439 & 441 & 366 & 352 & 307 & 233 & 78 & 64 \\
\hline 1024 & 4527 & 512 & 10 & 506 & 464 & 489 & 952 & 946 & 857 & 824913 & 897 & 733 & 688 & 942 & 942 & 862 & 848 & 788 & 687 & 510 & 496 \\
\hline
\end{tabular}

Table 6. LindnerPeikert with $\mathbf{s}_{(i)} \leftarrow_{\$}\{0,1\}$

\begin{tabular}{|c|c|c|c|c|c|c|c|c|c|c|c|c|c|c|c|c|c|c|c|c|c|c|}
\hline \multirow[b]{2}{*}{$n$} & \multicolumn{3}{|c|}{ MitM } & \multicolumn{3}{|c|}{ BKW } & \multicolumn{3}{|c|}{ SIS } & \multicolumn{4}{|c|}{ DEC } & & \multicolumn{3}{|c|}{ Kannan } & \multicolumn{5}{|c|}{ Bai-Gal } \\
\hline & 80 & & & & & $N_{\mathrm{S}}$ & & & & & & . & & & 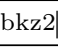 & & & & | & & $L_{\mathbf{s}, \lambda}$ & \\
\hline 64 & 45 & 32 & 6 & 53 & 42 & 37 & 31 & 46 & 9 & $\mid 0$ & 32 & 16 & 10 & 0 & 34 & 49 & 13 & 0 & 34 & 49 & 11 & 0 \\
\hline 128 & 78 & 64 & 7 & 86 & 74 & 68 & 34 & 49 & 10 & 0 & 36 & 20 & 11 & 0 & 37 & 52 & 14 & 0 & 37 & 52 & 12 & 0 \\
\hline 256 & 143 & 128 & 8 & 148 & 136 & 129 & 37 & 52 & 11 & 0 & 39 & 23 & 12 & 0 & 40 & 56 & 15 & 0 & 40 & 55 & 13 & 0 \\
\hline 512 & 272 & 256 & 9 & 272 & 260 & 252 & 40 & 55 & 12 & 0 & 42 & 26 & 13 & 0 & 44 & 59 & 16 & 0 & 43 & 58 & 14 & 0 \\
\hline 1024 & 529 & 512 & 10 & 520 & 507 & 498 & 69 & 79 & 13 & 0 & 66 & 50 & 14 & 0 & 67 & 78 & 17 & 0 & 59 & 78 & 15 & 0 \\
\hline 2048 & 1042 & 1024 & 11 & $\mid 1015$ & 1003 & 993 & 214 & 162 & 20 & 0 & 200 & 184 & 15 & 0 & 205 & 156 & 18 & 0 & 173 & 141 & 16 & 0 \\
\hline
\end{tabular}

Table 7. FHE with $L=2$ with $\mathbf{s}_{(i)} \leftarrow \$\{0,1\}$

small secret (cf. Section 6.3). This is because their parameters were chosen assuming Lindner and Peikert's estimate for the runtime of BKZ, which we rule out from among the choices of estimates because it implies a subexponential algorithm for solving LWE.

No single best algorithm. Our results indicate that there is not one algorithm which always outperforms all others on the parameter sets we tested, and so we cannot recommend to consider one particular algorithm to achieve security level $\lambda$. Which algorithm performs best depends on the concrete parameters considered. For small $n$, DEC may be favourable (see e.g. Table 4). For large $n$, BKW may be fastest when considering public-key encryption (see e.g. Table 3) but not when considering homomorphic encryption schemes which require large $q$ (cf. Tables 7 and 8).

We note that while the Arora-Ge algorithm and its Gröbner basis variants always perform much worse than other algorithms in our tests, it is shown in [ACFP14] that this family of algorithms outperforms other families when considering a particular variant of LWE, i.e. UniformNoise-LWE instances.

Finally, we note that all families of algorithms discussed in this work permit parallelisation. For BKW we can distribute (partial) elimination tables across computing units and for the Arora-GB algorithm we can rely on parallelised linear algebra routines. Lattice reduction, too, can be easily distributed for the instances considered in this work, as we are running in the low advantage regime, i.e. we are computing many independent lattice reductions on fresh or re-randomised inputs.

Time-memory trade-offs. According to our estimates of running BKZ, using sieving as the SVP oracle is faster than enumeration for large $n$. While this is to be expected given that sieving is asymptotically faster than enumeration, it might be surprising to see the crossover already for dimension $n=256$ in some cases. It is important to note, however, that sieving would require an amount of memory so substantial that, for most parameters we consider, it is not clear that sieving is worth considering even where it is faster "on paper". A completely analogous statement can be made when considering the BKW algorithm or the Arora-Ge and its Gröbner basis variant. Indeed, all algorithms which achieve a time complexity of $2^{\mathcal{O}(n)}$ also require memory of the order of $2^{\mathcal{O}(n)}$. An interesting open question is hence if an algorithm exists which solves LWE in $2^{\mathcal{O}(n)}$ operations but requiring only poly $(n)$ memory. 


\begin{tabular}{|c|c|c|c|c|c|c|c|c|c|c|c|c|c|c|c|c|c|c|c|c|c|}
\hline & | & \multicolumn{2}{|c|}{ MitM } & \multicolumn{3}{|c|}{ BKW } & \multicolumn{3}{|c|}{ SIS } & \multicolumn{4}{|c|}{ DEC } & \multicolumn{4}{|c|}{ Kannan } & \multicolumn{4}{|c|}{ Bai-Gal } \\
\hline$n$ & & & & & & & & & & & & & & & & & & & & & $\mathrm{~s} \gamma$ \\
\hline 512 & 274 & 256 & 9 & 287 & 270 & & 40 & 55 & 2 & $|0|$ & 4 & 28 & 13 & 0 & 43 & 58 & & $0 \mid$ & 43 & 58 & 14 \\
\hline 1024 & 531 & 512 & 10 & 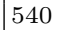 & 523 & 51 & 4 & $5 \xi$ & P & 0 & 4 & 30 & 14 & 0 & 47 & 6 & 6 & 0 & 46 & 62 & 15 \\
\hline 2048 & 1044 & 1024 & 11 & - & - & - & 46 & 61 & 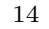 & 0 & 50 & 35 & 15 & 0 & 50 & 6 & 7 & 0 & 50 & 65 & 16 \\
\hline 4096 & 2069 & 2048 & 12 & - & - & - & 49 & 64 & 15 & 0 & 52 & 36 & 16 & 0 & 53 & 68 & 18 & 0 & 53 & 68 & 17 \\
\hline 8192 & 4118 & 4096 & 13 & - & - & - & 105 & 106 & 16 & 0 & 11 & 98 & 17 & 0 & 107 & 108 & 19 & 0 & 104 & 106 & 18 \\
\hline 1638 & 8215 & 8192 & 14 & - & - & - & 328 & 213 & 17 & 0 & 332 & 316 & 18 & 0 & 326 & 214 & 20 & 0 & 315 & 210 & 19 \\
\hline
\end{tabular}

Table 8. FHE with $L=10$ with $\mathbf{s}_{(i)} \leftarrow \$\{0,1\}$

Incomplete data. For, say, the decoding approach we are able to trade running time for success probability. On the other hand, we do not know how to do this when using Kannan embedding. As highlighted in Section 5.5, for a success probability of 0.1 we have that $\tau^{\prime} \approx 0.3$ is a fair estimate based on experiments in the literature, but no data is publicly available from which to estimate $\tau^{\prime}$ for smaller success probabilities. While the decoding approach seems to outperform the application of Kannan embedding as highlighted in Example 4, by a similar argument, it is to be expected that the algorithm of Bai and Galbraith could be shown to produce better results if such data was available.

This is just one area in which more data is required. Our estimator is built from the curves fitted to the data from the literature given in Table 1 and as such more experimental data on the runtime of enumeration and sieving would allow to refine these estimates. To reiterate, the analysis on which the estimator is based is sound given the current state of the art, but intrinsically depends on the formulae for sieving and enumeration, and so refinements in this area will refine our estimator accordingly. As lattice reduction is a central step in many of the algorithms, this is of particular importance.

Acknowledgements. Albrecht was supported by EPSRC grant EP/L018543/1 "Multilinear Maps in Cryptography". Player was supported by an ACE-CSR PhD grant. Scott was supported by EPSRC grant EP/K035584/1. We thank Steven Galbraith and Paul Kirchner for pointing out mistakes in an earlier version of this work. 


\section{References}

$\mathrm{ACF}^{+}$15. Martin R. Albrecht, Carlos Cid, Jean-Charles Faugère, Robert Fitzpatrick, and Ludovic Perret. On the complexity of the BKW algorithm on LWE. Designs, Codes and Cryptography, 74:325-354, 2015.

ACFP14. Martin R. Albrecht, Carlos Cid, Jean-Charles Faugère, and Ludovic Perret. Algebraic algorithms for LWE. Cryptology ePrint Archive, Report 2014/1018, 2014. http://eprint.iacr . org/2014/1018.

ACPS09. Benny Applebaum, David Cash, Chris Peikert, and Amit Sahai. Fast cryptographic primitives and circular-secure encryption based on hard learning problems. In Halevi [Hal09], pages 595618.

ACPS14. Martin Albrecht, David Cadé, Xavier Pujol, and Damien Stehlé. fplll, development version. Available at https://github.com/dstehle/fplll, 2014.

$\mathrm{ADH}^{+}$19. Martin R. Albrecht, Léo Ducas, Gottfried Herold, Elena Kirshanova, Eamonn W. Postlethwaite, and Marc Stevens. The general sieve kernel and new records in lattice reduction. In Yuval Ishai and Vincent Rijmen, editors, EUROCRYPT 2019, Part II, volume 11477 of LNCS, pages 717-746. Springer, Heidelberg, May 2019.

ADPS16. Erdem Alkim, Léo Ducas, Thomas Pöppelmann, and Peter Schwabe. Post-quantum key exchange - A new hope. In Thorsten Holz and Stefan Savage, editors, USENIX Security 2016, pages 327-343. USENIX Association, August 2016.

ADRS14. Divesh Aggarwal, Daniel Dadush, Oded Regev, and Noah Stephens-Davidowitz. Solving the shortest vector problem in $2^{n}$ time via discrete gaussian sampling. CoRR, abs/1412.7994, 2014.

$\mathrm{AFC}^{+}$13. Martin R. Albrecht, Robert Fitzpatrick, Daniel Cabracas, Florian Göpfert, and Michael Schneider. A generator for LWE and Ring-LWE instances, 2013. available at http: //www.iacr.org/news/files/2013-04-29lwe-generator.pdf.

AFFP14. Martin R. Albrecht, Jean-Charles Faugère, Robert Fitzpatrick, and Ludovic Perret. Lazy modulus switching for the BKW algorithm on LWE. In Hugo Krawczyk, editor, PKC 2014, volume 8383 of LNCS, pages 429-445. Springer, Heidelberg, March 2014.

AFG14. Martin R. Albrecht, Robert Fitzpatrick, and Florian Göpfert. On the efficacy of solving LWE by reduction to unique-SVP. In Hyang-Sook Lee and Dong-Guk Han, editors, ICISC 13, volume 8565 of LNCS, pages 293-310. Springer, Heidelberg, November 2014.

AG11. Sanjeev Arora and Rong Ge. New algorithms for learning in presence of errors. In Luca Aceto, Monika Henzinger, and Jiri Sgall, editors, ICALP 2011, Part I, volume 6755 of LNCS, pages 403-415. Springer, Heidelberg, July 2011.

AGVW17. Martin R. Albrecht, Florian Göpfert, Fernando Virdia, and Thomas Wunderer. Revisiting the expected cost of solving uSVP and applications to LWE. In Takagi and Peyrin [TP17], pages 297-322.

Ajt96. Miklós Ajtai. Generating hard instances of lattice problems (extended abstract). In 28th ACM STOC, pages 99-108. ACM Press, May 1996.

Alb13. Martin Albrecht. BKW-LWE, 2013. https://bitbucket.org/malb/bkw-lwe/.

Alb17. Martin R. Albrecht. On dual lattice attacks against small-secret LWE and parameter choices in HElib and SEAL. In Jean-Sébastien Coron and Jesper Buus Nielsen, editors, EUROCRYPT 2017, Part II, volume 10211 of LNCS, pages 103-129. Springer, Heidelberg, April / May 2017.

Bab85. László Babai. On Lovász' lattice reduction and the nearest lattice point problem (shortened version). In Kurt Mehlhorn, editor, STACS '86, volume 82 of Lecture Notes in Computer Science, pages 13-20. Springer, 1985.

BBD09. Daniel J. Bernstein, Johannes Buchmann, and Erik Dahmen, editors. Post-Quantum Cryptography. Springer, 2009.

BBGS17. Nina Bindel, Johannes Buchmann, Florian Göpfert, and Markus Schmidt. Estimation of the hardness of the learning with errors problem with a restricted number of samples. Cryptology ePrint Archive, Report 2017/140, 2017. https://eprint.iacr.org/2017/140.

BCS97. Peter Bürgisser, Michael Clausen, and Mohammad Amin Shokrollahi. Algebraic Complexity Theory, volume 315 of Grundlehren der mathematischen Wissenschaften. Springer, 1997.

BDGL16. Anja Becker, Léo Ducas, Nicolas Gama, and Thijs Laarhoven. New directions in nearest neighbor searching with applications to lattice sieving. In Robert Krauthgamer, editor, 27th SODA, pages 10-24. ACM-SIAM, January 2016. 
BG14. Shi Bai and Steven D. Galbraith. Lattice decoding attacks on binary LWE. In Willy Susilo and Yi Mu, editors, ACISP 14, volume 8544 of LNCS, pages 322-337. Springer, Heidelberg, July 2014.

BGV12. Zvika Brakerski, Craig Gentry, and Vinod Vaikuntanathan. (Leveled) fully homomorphic encryption without bootstrapping. In Shafi Goldwasser, editor, ITCS 2012, pages 309-325. ACM, January 2012.

BKW03. Avrim Blum, Adam Kalai, and Hal Wasserman. Noise-tolerant learning, the parity problem, and the statistical query model. Journal of the ACM, 50(4):506-519, July 2003.

$\mathrm{BLP}^{+}$13. Zvika Brakerski, Adeline Langlois, Chris Peikert, Oded Regev, and Damien Stehlé. Classical hardness of learning with errors. In Dan Boneh, Tim Roughgarden, and Joan Feigenbaum, editors, 45th ACM STOC, pages 575-584. ACM Press, June 2013.

BV11. Zvika Brakerski and Vinod Vaikuntanathan. Efficient fully homomorphic encryption from (standard) LWE. In Rafail Ostrovsky, editor, 52nd FOCS, pages 97-106. IEEE Computer Society Press, October 2011.

Che52. Herman Chernoff. A measure of asymptotic efficiency for tests of a hypothesis based on the sum of observations. The Annals of Mathematical Statistics, pages 493-507, 1952.

Che13. Yuanmi Chen. Réduction de réseau et sécurité concrète du chiffrement complètement homomorphe. PhD thesis, Paris 7, 2013.

CHHS19. Jung Hee Cheon, Minki Hhan, Seungwan Hong, and Yongha Son. A hybrid of dual and meet-in-the-middle attack on sparse and ternary secret LWE. IEEE Access, 7:89497-89506, 2019.

CHKP12. David Cash, Dennis Hofheinz, Eike Kiltz, and Chris Peikert. Bonsai trees, or how to delegate a lattice basis. Journal of Cryptology, 25(4):601-639, October 2012.

CN11. Yuanmi Chen and Phong Q. Nguyen. BKZ 2.0: Better lattice security estimates. In Dong Hoon Lee and Xiaoyun Wang, editors, ASIACRYPT 2011, volume 7073 of LNCS, pages 1-20. Springer, Heidelberg, December 2011.

CN12. Yuanmi Chen and Phong Q. Nguyen. BKZ 2.0: Better lattice security estimates (full version). http://www.di.ens.fr/ ychen/research/Full_BKZ.pdf, 2012.

CPS13. David Cadé, Xavier Pujol, and Damien Stehlé. fpLLL 4.0.4, 2013. http://perso.ens-lyon. $\mathrm{fr} /$ damien.stehle/fplll/.

DB13. Léo Ducas-Binda. Signatures Fondées sur les Réseaux Euclidiens: Attaques, Analyses et Optimisations. PhD thesis, École Normale Supérieure Paris, 2013. http://cseweb.ucsd.edu/ $\sim$ lducas/Thesis/index.html.

DTV15. Alexandre Duc, Florian Tramèr, and Serge Vaudenay. Better algorithms for LWE and LWR. Cryptology ePrint Archive, Report 2015/056, 2015. http://eprint.iacr.org/2015/056.

Duc18. Léo Ducas. Shortest vector from lattice sieving: A few dimensions for free. In Jesper Buus Nielsen and Vincent Rijmen, editors, EUROCRYPT 2018, Part I, volume 10820 of LNCS, pages 125-145. Springer, Heidelberg, April / May 2018.

$\mathrm{EHK}^{+}$18. $\quad$ Andre Esser, Felix Heuer, Robert Kübler, Alexander May, and Christian Sohler. DissectionBKW. In Hovav Shacham and Alexandra Boldyreva, editors, CRYPTO 2018, Part II, volume 10992 of $L N C S$, pages 638-666. Springer, Heidelberg, August 2018.

Gen09. Craig Gentry. A fully homomorphic encryption scheme. PhD thesis, Stanford University, 2009. crypto.stanford.edu/craig.

GGH13. Sanjam Garg, Craig Gentry, and Shai Halevi. Candidate multilinear maps from ideal lattices. In Thomas Johansson and Phong Q. Nguyen, editors, EUROCRYPT 2013, volume 7881 of LNCS, pages 1-17. Springer, Heidelberg, May 2013.

GGH14. Craig Gentry, Sergey Gorbunov, and Shai Halevi. Graph-induced multilinear maps from lattices. Cryptology ePrint Archive, Report 2014/645, 2014. http://eprint.iacr.org/2014/ 645.

GHS12a. Craig Gentry, Shai Halevi, and Nigel P. Smart. Fully homomorphic encryption with polylog overhead. In David Pointcheval and Thomas Johansson, editors, EUROCRYPT 2012, volume 7237 of LNCS, pages 465-482. Springer, Heidelberg, April 2012.

GHS12b. Craig Gentry, Shai Halevi, and Nigel P. Smart. Homomorphic evaluation of the AES circuit. In Reihaneh Safavi-Naini and Ran Canetti, editors, CRYPTO 2012, volume 7417 of $L N C S$, pages 850-867. Springer, Heidelberg, August 2012.

GHS12c. Craig Gentry, Shai Halevi, and Nigel P. Smart. Homomorphic evaluation of the AES circuit. Cryptology ePrint Archive, Report 2012/099, 2012. http://eprint.iacr.org/2012/099.

GJMS17. Qian Guo, Thomas Johansson, Erik Mårtensson, and Paul Stankovski. Coded-BKW with sieving. In Takagi and Peyrin [TP17], pages 323-346. 
GJS15. Qian Guo, Thomas Johansson, and Paul Stankovski. Coded-BKW: Solving LWE using lattice codes. In Gennaro and Robshaw [GR15], pages 23-42.

GKPV10. Shafi Goldwasser, Yael Tauman Kalai, Chris Peikert, and Vinod Vaikuntanathan. Robustness of the learning with errors assumption. In Andrew Chi-Chih Yao, editor, ICS, pages 230-240. Tsinghua University Press, 2010.

GN08. Nicolas Gama and Phong Q. Nguyen. Predicting lattice reduction. In Nigel P. Smart, editor, EUROCRYPT 2008, volume 4965 of LNCS, pages 31-51. Springer, Heidelberg, April 2008.

GNR10. Nicolas Gama, Phong Q. Nguyen, and Oded Regev. Lattice enumeration using extreme pruning. In Henri Gilbert, editor, EUROCRYPT 2010, volume 6110 of LNCS, pages 257-278. Springer, Heidelberg, May / June 2010.

GPV08. Craig Gentry, Chris Peikert, and Vinod Vaikuntanathan. Trapdoors for hard lattices and new cryptographic constructions. In Richard E. Ladner and Cynthia Dwork, editors, 40th ACM STOC, pages 197-206. ACM Press, May 2008.

GR15. Rosario Gennaro and Matthew J. B. Robshaw, editors. CRYPTO 2015, Part I, volume 9215 of LNCS. Springer, Heidelberg, August 2015.

Hal09. Shai Halevi, editor. CRYPTO 2009, volume 5677 of LNCS. Springer, Heidelberg, August 2009.

HJP14. William Hart, Fredrik Johansson, and Sebastian Pancratz. FLINT: Fast Library for Number Theory, 2014. Version 2.4.4, http://flintlib.org.

HPS11a. Guillaume Hanrot, Xavier Pujol, and Damien Stehlé. Analyzing blockwise lattice algorithms using dynamical systems. In Phillip Rogaway, editor, CRYPTO 2011, volume 6841 of LNCS, pages 447-464. Springer, Heidelberg, August 2011.

HPS11b. Guillaume Hanrot, Xavier Pujol, and Damien Stehlé. Algorithms for the shortest and closest lattice vector problems. In Coding and Cryptology, volume 6639 of Lecture Notes in Computer Science, pages 159-190. Springer, 2011.

$\mathrm{JOP}^{+}$. $\quad$ Eric Jones, Travis Oliphant, Pearu Peterson, et al. SciPy: Open source scientific tools for Python, 2001-. [Online; accessed 2015-09-02].

Jou09. Antoine Joux. Algorithmic Cryptanalysis. Chapman \& Hall/CRC, 1st edition, 2009.

Kan87. Ravi Kannan. Minkowski's convex body theorem and integer programming. Math. Oper. Res., 12(3):415-440, August 1987.

KF15. Paul Kirchner and Pierre-Alain Fouque. An improved BKW algorithm for LWE with applications to cryptography and lattices. In Gennaro and Robshaw [GR15], pages 43-62.

$\mathrm{KMR}^{+}$94. Michael J. Kearns, Yishay Mansour, Dana Ron, Ronitt Rubinfeld, Robert E. Schapire, and Linda Sellie. On the learnability of discrete distributions. In 26th ACM STOC, pages 273-282. ACM Press, May 1994.

Laa14. Thijs Laarhoven. Sieving for shortest vectors in lattices using angular locality-sensitive hashing. Cryptology ePrint Archive, Report 2014/744, 2014. http://eprint.iacr.org/2014/744.

Laa15. Thijs Laarhoven. Search problems in cryptography: from fingerprinting to lattice sieving. PhD thesis, Technische Universiteit Einhoven, 2015.

LdW15. Thijs Laarhoven and Benne de Weger. Faster sieving for shortest lattice vectors using spherical locality-sensitive hashing. Cryptology ePrint Archive, Report 2015/211, 2015. http: //eprint.iacr.org/2015/211.

LLL82. A.K. Lenstra, Jr. Lenstra, H.W., and L. Lovász. Factoring polynomials with rational coefficients. Mathematische Annalen, 261(4):515-534, 1982.

LLLS11. Cong Ling, Shuiyin Liu, Laura Luzzi, and Damien Stehlé. Decoding by embedding: Correct decoding radius and DMT optimality. In Alexander Kuleshov, Vladimir Blinovsky, and Anthony Ephremides, editors, 2011 IEEE International Symposium on Information Theory Proceedings, ISIT 2011, St. Petersburg, Russia, July 31 - August 5, 2011, pages 1106-1110. IEEE, 2011.

LM09. Vadim Lyubashevsky and Daniele Micciancio. On bounded distance decoding, unique shortest vectors, and the minimum distance problem. In Halevi [Hal09], pages 577-594.

LMvdP15. Thijs Laarhoven, Michele Mosca, and Joop van de Pol. Finding shortest lattice vectors faster using quantum search. Designs, Codes and Cryptography, 77(2):375-400, Dec 2015.

LN13. Mingjie Liu and Phong Q. Nguyen. Solving BDD by enumeration: An update. In Ed Dawson, editor, CT-RSA 2013, volume 7779 of LNCS, pages 293-309. Springer, Heidelberg, February / March 2013.

LN14. Tancrède Lepoint and Michael Naehrig. A comparison of the homomorphic encryption schemes FV and YASHE. In David Pointcheval and Damien Vergnaud, editors, AFRICACRYPT 14, volume 8469 of LNCS, pages 318-335. Springer, Heidelberg, May 2014. 
Lov86. László Lovász. An algorithmic theory of numbers, graphs and convexity. CBMS-NSF regional conference series in applied mathematics. Philadelphia, Pa. Society for Industrial and Applied Mathematics, 1986.

LP11. Richard Lindner and Chris Peikert. Better key sizes (and attacks) for LWE-based encryption. In Aggelos Kiayias, editor, CT-RSA 2011, volume 6558 of LNCS, pages 319-339. Springer, Heidelberg, February 2011.

MGH80. J. J. Moré, B. S. Garbow, and K. E. Hillstrom. User Guide for MINPACK-1. ANL-80-74, Argonne National Laboratory, 1980.

MP13. Daniele Micciancio and Chris Peikert. Hardness of SIS and LWE with small parameters. In Ran Canetti and Juan A. Garay, editors, CRYPTO 2013, Part I, volume 8042 of LNCS, pages 21-39. Springer, Heidelberg, August 2013.

MR09. Daniele Micciancio and Oded Regev. Lattice-based cryptography. In Bernstein et al. [BBD09], pages $147-191$.

MW15. Daniele Micciancio and Michael Walter. Fast lattice point enumeration with minimal overhead. In Piotr Indyk, editor, 26th SODA, pages 276-294. ACM-SIAM, January 2015.

Ngu10. Phong Q. Nguyen. Hermite's constant and lattice algorithms. In Nguyen and Vallée [NV10], pages 19-69.

Ngu11. Phong Q. Nguyen. Lattice reduction algorithms: Theory and practice (invited talk). In Kenneth G. Paterson, editor, EUROCRYPT 2011, volume 6632 of LNCS, pages 2-6. Springer, Heidelberg, May 2011.

NS05. Phong Q. Nguyen and Damien Stehlé. Floating-point LLL revisited. In Ronald Cramer, editor, EUROCRYPT 2005, volume 3494 of LNCS, pages 215-233. Springer, Heidelberg, May 2005.

NV10. Phong Q. Nguyen and Brigitte Vallée, editors. The LLL Algorithm - Survey and Applications. Information Security and Cryptography. Springer, 2010.

Pei09. Chris Peikert. Public-key cryptosystems from the worst-case shortest vector problem: extended abstract. In Michael Mitzenmacher, editor, 41st ACM STOC, pages 333-342. ACM Press, May / June 2009.

Per14. Clèment Pernet. High performance and reliable algebraic computing. Symbolic Computation. Université Joseph Fourier, Grenoble 1, 2014.

Pla18. Rachel Player. Parameter selection in lattice-based cryptography. PhD thesis, Royal Holloway, University of London, 2018.

PVW08. Chris Peikert, Vinod Vaikuntanathan, and Brent Waters. A framework for efficient and composable oblivious transfer. In David Wagner, editor, CRYPTO 2008, volume 5157 of LNCS, pages 554-571. Springer, Heidelberg, August 2008.

PW11. Chris Peikert and Brent Waters. Lossy trapdoor functions and their applications. SIAM J. Comput., 40(6):1803-1844, 2011.

Reg05. Oded Regev. On lattices, learning with errors, random linear codes, and cryptography. In Harold N. Gabow and Ronald Fagin, editors, 37th ACM STOC, pages 84-93. ACM Press, May 2005.

Reg09. Oded Regev. On lattices, learning with errors, random linear codes, and cryptography. Journal of the ACM, 56(6):34:1-34:40, September 2009.

Reg10. Oded Regev. The learning with errors problem (invited survey). In IEEE Conference on Computational Complexity, pages 191-204. IEEE Computer Society, 2010.

$\mathrm{S}^{+}$14. William Stein et al. Sage Mathematics Software Version 6.3. The Sage Development Team, 2014. http://www. sagemath.org.

Sch03. Claus Peter Schnorr. Lattice reduction by random sampling and birthday methods. In STACS, volume 2607 of Lecture Notes in Computer Science, pages 145-156. Springer, 2003.

SE94. Claus Peter Schnorr and M. Euchner. Lattice basis reduction: Improved practical algorithms and solving subset sum problems. Math. Program., 66:181-199, 1994.

Sho. V. Shoup. Number Theory Library 5.5.2 (NTL) for C++. http://www. shoup.net/ntl/.

Ste13. Damien Stehlé. An overview of lattice reduction algorithms. Invited talk at ICISC, 2013.

TP17. Tsuyoshi Takagi and Thomas Peyrin, editors. ASIACRYPT 2017, Part I, volume 10624 of LNCS. Springer, Heidelberg, December 2017.

vDGHV10. Marten van Dijk, Craig Gentry, Shai Halevi, and Vinod Vaikuntanathan. Fully homomorphic encryption over the integers. In Henri Gilbert, editor, Advances in Cryptology - EUROCRYPT 2010, 29th Annual International Conference on the Theory and Applications of Cryptographic Techniques, Monaco / French Riviera, May 30 - June 3, 2010. Proceedings, volume 6110 of Lecture Notes in Computer Science, pages 24-43. Springer, 2010. 
vdPS13. Joop van de Pol and Nigel P. Smart. Estimating key sizes for high dimensional lattice-based systems. In Martijn Stam, editor, 14th IMA International Conference on Cryptography and Coding, volume 8308 of LNCS, pages 290-303. Springer, Heidelberg, December 2013.

Wal14. Michael Walter. Lattice point enumeration on block reduced bases. Cryptology ePrint Archive, Report 2014/948, 2014. http://eprint.iacr.org/2014/948. 